\title{
Spectroscopy of Globular Clusters in M81 ${ }^{1}$
}

\author{
Linda L. Schroder \\ Lick Observatory, University of California, Santa Cruz, CA 95064 \\ Electronic mail: linda@ucolick.org \\ Jean P. Brodie \\ Lick Observatory, University of California, Santa Cruz, CA 95064 \\ Electronic mail: brodie@lick.ucsc.edu
}

\author{
Markus Kissler-Patig \\ European Southern Observatory, Karl-Schwarzschild-Str.2 85748 Garching, Germany \\ Electronic mail: mkissler@eso.org \\ John P. Huchra \\ Harvard-Smithsonian Center for Astrophysics, 60 Garden St, Cambridge MA 01238 \\ Electronic mail: huchra@cfa.harvard.edu
}

Andrew C. Phillips

Lick Observatory, University of California, Santa Cruz, CA 95064

Electronic mail: phillips@ucolick.org

\begin{abstract}
We present moderate-resolution spectroscopy of globular clusters around the $\mathrm{Sa} / \mathrm{Sb}$ spiral galaxy M81 (NGC 3031). Sixteen candidate clusters were observed with the Low Resolution Imaging Spectrograph on the Keck I telescope. All are confirmed as bona fide globular clusters, although one of the clusters appears to have been undergoing a transient event during our observations. In general, the M81 globular cluster system is found to be very similar to the Milky Way and M31 systems, both chemically and kinematically. A kinematic analysis of the velocities of $44 \mathrm{M} 81$ globular clusters, (the 16 presented here and 28 from previous work) strongly suggests that the red, metal-rich clusters are rotating in the same sense as the gas in the disk of M81. The blue, metal-poor clusters have halo-like kinematics, showing no evidence for rotation. The kinematics of clusters whose projected galactocentric radii lie between 4 and 8 kpc suggest that they are rotating much more than those with projected galactocentric radii outside these bounds. We suggest that these rotating, intermediate-distance clusters are analogous to the kinematic subpopulation in the metal-rich, disk globular clusters observed in the Milky Way and we present evidence for the existence of a similar sub-population in the metal-rich clusters of M31. With one exception, all of the M81 clusters in our sample have ages that are consistent with Milky Way and M31 globular clusters. One cluster may be as young as a few Gyrs. The correlations between absorption-line indices established for Milky Way and M31 globular clusters also hold in the M81 cluster system, at least at the upper end of the metallicity distribution (which our sample probes). On the whole, the mean metallicity of the M81 globular cluster system is similar to the metallicity of the Milky Way and M31 globular cluster systems. To within a factor of two, the projected mass of M81 is similar to the massses of the Milky Way and M31. Its mass profile indicates the presence of a dark matter halo.
\end{abstract}

Subject headings: galaxies: spiral, galaxies: individual (M81,NGC3031,M31), globular clusters: general 


\section{Introduction}

Globular clusters are ideal probes of the dynamical properties and chemical histories of their host galaxies. They are bright enough to be observed at large distances, old enough to have been "witnesses" to the processes by which galaxies form and evolve, and are found in all types of galaxies. For all but the nearest galaxies, globular clusters are the sole providers of information about the stellar population in galaxy halos.

Chemically and kinematically distinct subsystems have been established in the globular cluster systems of both the Milky Way and our nearest spiral neighbor, M31. The presence of such subsystems places significant constraints on spiral galaxy formation theories and may also bear on understanding the formation and evolution of elliptical galaxies. It has been suggested (Schweizer 1986) that some elliptical galaxies may form via the merging of spirals. In a gaseous merger, the resulting globular cluster system would be expected to contain any globular clusters originally associated with the merger participants and perhaps new clusters formed during the merger (Ashman \& Zepf 1992). While study of globular cluster systems around ellipticals is important for validating or refuting the merger hypothesis, interpreting such studies requires understanding the natural variations in the observed properties of globular cluster systems around spirals. Key properties such as age and metallicity are most reliably constrained through spectroscopy, while kinematic and individual element abundance information are obtained exclusively so. The advent of 10-meter class telescopes and multi-object spectrographs has made progress on this front feasible.

The Milky Way globular cluster system is naturally the benchmark by which the systems of other spirals are measured. One of its most striking features is its bimodal metallicity distribution, with peaks at $[\mathrm{Fe} / \mathrm{H}]=-1.6$ and -0.5 (Zinn 1985; Armandroff \& Zinn 1988; Armandroff 1989). Furthermore, these metallicity sub-populations are

\footnotetext{
${ }^{1}$ Data presented herein were obtained at the W.M. Keck Observatory, which is operated as a scientific partnership among the California Institute of Technology, the University of California and the National Aeronautics and Space Administration. The Observatory was made possible by the generous financial support of the W.M. Keck Foundation.
}

spatially and kinematically distinct (Zinn 1985; Hesser, Shawl \& Meyer 1986; Armandroff \& Zinn 1988; Armandroff 1989). The metal-rich clusters comprise a centrally-concentrated, flattened, rotating system with a low velocity dispersion, referred to by Zinn (1985) as the disk population. Armandroff (1989) associated these disk clusters with the Milky Way's thick-disk component, based on similar spatial, kinematic and chemical properties of the two systems. More recently, it has been reported that the properties of the metal-rich clusters lying within $3 \mathrm{kpc}$ of the Galactic center have more in common with the bulge than with the thick disk (Minniti 1995; Cote 1999). Burkert \& Smith (1997) further explored sub-structures in the metal-rich globular cluster population, identifying a group belonging to the galactic bar and one comprising a ring of rapidly-rotating clusters with a low velocity dispersion and galactocentric radii between 4 and $6 \mathrm{kpc}$ (these are the thick-disk clusters of Armandroff (1989)). To first order, Zinn (1985) found the properties of the Milky Way's metal-poor globular clusters to be well matched with those of the halo field stars: they are spherically distributed, rotate slowly and have a large line-of-sight velocity dispersion. However, Zinn (1993) reported substructure in the Milky Way's halo clusters as well.

The M31 globular cluster system was the first system around another spiral to be studied in detail. A number of imaging programs resulted in several catalogues and refined lists of globular cluster candidates (Crampton et al. 1985; Battistini, et al. 1980, 1987; Battistini et al. 1993; Reed, Harris \& Harris 1994). Insight into the chemical and kinematic nature of M31's globular cluster system resulted from the optical spectroscopy studies of Huchra, Stauffer \& Van Speybroeck (1982), Kent, Huchra \& Stauffer (1989), Huchra, Brodie \& Kent (1991) and Brodie \& Huchra (1991). All came to the same conclusion: the globular cluster system of M31 is, for the most part, very similar to the Milky Way system. Huchra, Brodie \& Kent (1991) derived metallicities for 149 clusters and found them to be consistent with the metallicity distribution of the Milky Way globular cluster system, but they were unable to distinguish any obvious bimodality. They noted that large uncertainties in their individual metallicity measurements could be obscuring its presence. Ashman \& Bird 
(1993) subsequently performed a statistical analysis on the Huchra, Brodie \& Kent (1991) data and found quantitative evidence for a bimodal metallicity distribution at the $98.4 \%$ confidence level. New optical and infrared color distributions by Barmby et al. (2000) definitively support the presence of bimodality.

Based on velocities of 60 clusters, Huchra, Stauffer \& Van Speybroeck (1982) detected a modest, but significant rotation for the entire system. Subsequent studies have yielded evidence for kinematic sub-systems as well. Huchra, Brodie \& Kent (1991) reported that the metal-rich clusters in the inner few kpc of M31 are rapidly rotating, while at larger radii they observed little rotation in the M31 globular clusters, regardless of cluster metallicity. Barmby et al. (2000) recently repeated the kinematic analysis of Huchra, Brodie \& Kent (1991) with an additional 74 clusters and derived the same result.

As similar to the Milky Way system as M31's globular cluster system appears, there do seem to be some genuine differences. The M31 clusters contain, on average, a higher abundance of nitrogen than Milky Way globular clusters. This result was first reported as a CN enhancement in the spectroscopic studies of Burstein et al. (1984) and Brodie \& Huchra (1991) and more recently in the ultraviolet spectroscopy of Ponder et al. (1998), who established it as a nitrogen overabundance. Brodie \& Huchra (1991) also reported evidence of calcium enhancement in the M31 cluster spectra. In addition, the M31 system contains a handful globular clusters which appear to be "young", based on the strength of their Balmer-line absorption (Sargent et al. 1977; Cowley \& Burstein 1988; Elson \& Walterbos 1988; Brodie \& Huchra 1991; Barmby et al. 2000). No such clusters have been observed in the Milky Way.

Globular cluster systems around a few other spirals have been examined to a more limited extent. Photometric (Christian \& Schommer 1982; Cohen, Persson \& Searle 1984; Sharov \& Lyutyj 1984; Christian \& Schommer 1988; Chandar et al. 1999a,b,c) and spectroscopic (Schommer et al. 1991; Brodie \& Huchra 1991) studies of M33, the late-type, low-luminosity, Local-Group spiral, have revealed that it contains a number of young and intermediate-age compact star clusters. In addition, it contains about 25 typical old globular clusters in a halo distribution with little net rotation.

Beyond the Local Group, M104 and M81 are the only spiral galaxies whose globular cluster systems have been studied spectroscopically. Velocities for 34 M104 globular clusters were obtained by Bridges et al. (1997), who found marginal evidence for rotation. The quality of their data was too poor to measure metallicities for individual clusters. They produced a rough estimate of the mean metallicity of the system from a composite spectrum of all 34 objects, finding $\langle[\mathrm{Fe} / \mathrm{H}]\rangle=$ $-0.7 \pm 0.3$. This value is substantially higher than the metallicity of the Milky Way and M31 globular cluster systems, but M104 is extremely bulgedominated. It is also significantly more luminous than the Milky Way or M31, and its mean globular cluster metallicity is similar to the values found for globular clusters around elliptical and giant elliptical galaxies with a luminosity similar to that of M104 (see Table 4 in Bridges et al. 1994.)

At a distance of 3.7 Mpc (Freedman et al. 1994) M81 is at roughly half the distance of M104. It is an Sa/Sb-type spiral, very similar to M31, and roughly as massive as the Milky Way. Its proximity and morphology make it a good candidate for reaching beyond Local Group toward a detailed, high signal-to-noise spectroscopic study of a spiral galaxy globular cluster system for comparison to the Milky Way and M31 systems. In their study of extragalactic globular clusters, Brodie \& Huchra (1991) derived spectroscopic metallicities for eight clusters in M81 and found a sample mean of $[\mathrm{Fe} / \mathrm{H}]=-1.46 \pm 0.31$. Perelmuter \& Racine (1995) conducted a photometric study of the M81 globular cluster system and compiled a catalogue of globular cluster candidates, using proper motion to minimize contamination from foreground stars. In a spectroscopic follow-up, Perelmuter, Brodie \& Huchra (1995) obtained low signal-tonoise spectra of 82 candidates, 25 of which were established as bona fide members of the M81 globular cluster system. The generally poor signal-tonoise of their data resulted in rather large uncertainties in the metallicities of individual clusters. They derived a more precise mean metallicity of their sample from a composite spectrum, finding $\langle[\mathrm{Fe} / \mathrm{H}]\rangle=-1.48 \pm 0.19$. Their analysis of the cluster velocities showed a hint of rotation in the M81 globular cluster system. 
In this work we extend the spectroscopic studies of Brodie \& Huchra (1991) and Perelmuter, Brodie \& Huchra (1995) with significantly higher signal-to-noise spectra of an additional 16 members of the M81 globular cluster system. Section 2 describes our observations and data reduction procedure. A discussion of the abundance ratios, mean metallicities and ages of the M81 clusters and a comparison of these properties to M31 and the Milky Way globular clusters can be found in Section 3. In Section 4, we combine our velocity data with the velocities of additional clusters from the work of Perelmuter, Brodie \& Huchra (1995) and Brodie \& Huchra (1991), and discuss the kinematics of the resulting sample. Section 5 contains a summary of our results and a brief discussion of their implications for using globular cluster systems as probes for studying galaxy formation and evolution.

\section{Observations and Data Reduction}

\subsection{Observations}

The targets were selected from the candidate list of Perelmuter \& Racine (1995). The specific candidates observed were selected on the basis of brightness and the desire to simultaneously observe the maximum possible number of candidates with the spectrograph in multi-object mode. Spectra of 16 globular cluster candidates were obtained on 1994 November 30 using the Low Resolution Imaging Spectrograph (LRIS) (Oke et al. 1995) on the Keck I 10-m telescope in $\sim 0$ " $^{\prime \prime}$ seeing. The candidates were observed through a single slit mask. Weather and technical problems prevented observations through two additional masks, so our sample is spatially biased toward one side of the galaxy. The $600 \mathrm{l} / \mathrm{mm}$ grating, blazed at $7500 \AA$, and slitlets of $1^{\prime \prime}$ width resulted in a dispersion of $1.25 \AA$ /pixel and a spectral resolution of $5-6 \AA$. Because the horizontal positions of the slitlets varied across the slit mask, the spectral range falling on the detector was different for each slitlet. The shortest wavelength observed was $3700 \AA$ and the longest was $7420 \AA$. Each candidate spectrum covers $2560 \AA$ between those two extremes. The region of common overlap for all candidates is 4680$6260 \AA$. We obtained $5 \times 900 \mathrm{~s}$ integrations for a total integration of $4500 \mathrm{~s}$. A complete list of the observed cluster candidates' positions, magni- tudes and colors is provided in Table 1 . Figure 1 shows the positions of the candidates with respect to the galaxy center, along with the positions of established clusters from the work of Perelmuter, Brodie \& Huchra (1995) and Brodie \& Huchra (1991).

\subsection{Data Reduction}

These data were reduced using the following procedure. A mean bias, assumed to be the mean level of the CCD overscan region, was subtracted from both science and calibration images. In addition, the small-scale bias structure was determined by combining multiple one-second dark exposures. This bias structure was subtracted from all other science and calibration images. After the images were cleaned of cosmic rays, a map of the small scale variations in detector sensitivity and grating efficiency was removed by fitting a fifth- or sixth-order polynomial to a halogen lamp spectrum taken through each slitlet in the mask and dividing the lamp spectra by the polynomial. The resulting normalized images were divided into the science images to remove the small scale variations.

Each slitlet was subsequently processed using the $\mathrm{IRAF}^{2}$ routines of the noao.twodspec package. Ident, reident and fitcoords were run on spectra of $\mathrm{Hg}, \mathrm{Ne}, \mathrm{Ar}$ and $\mathrm{Kr}$ lamps to determine the wavelength solution and then transform was used to apply the appropriate solution to the twodimensional spectra of the candidate objects. The object spectra were extracted using the IRAF routines of the noao.apextract package, employing the optimal spectral extraction scheme detailed by Horne (1986). The polynomial used to approximate the background was typically a linear fit. For a few candidates, however, proximity to end of the slitlet required approximating the background as a constant.

The extracted candidate spectra were fluxcalibrated using a long slit spectrum of the standard star EG145 taken on the same night as the candidate observations. Since its location in the

${ }^{2}$ IRAF is distributed by the National Optical Astronomy Observatories, which are operated by the Association of Universities for Research in Astronomy, Inc. under cooperative agreement with the National Science Foundation. 
dispersion direction on the mask determines what wavelength region falls onto the detector for an individual slitlet, the wavelength coverage for some candidate cluster spectra is not coincident with the coverage for the flux standard. The result is that the continuum shape is only accurately determined in the spectral region of $3900 \AA-6500 \AA$. None of the absorption features of interest lie beyond $6500 \AA$, while two spectral features of interest (see Section 3) require measurements below $3900 \AA$. The final spectra of all 16 candidates are shown in Figure 2. They have been corrected for foreground reddening in the direction of M81 using the IRAF task noao.onedspec.deredden with $\mathrm{E}_{(\mathrm{B}-\mathrm{V})}=0.1$ and $\mathrm{R}_{\mathrm{V}}=3.1$ (Burstein \& Heiles 1984), then shifted to the rest frame.

M81's low systemic heliocentric velocity $\left(-34 \mathrm{~km} \mathrm{~s}^{-1}\right)$ precludes the establishment of globular cluster candidates as bona fide M81 globular clusters on the basis of radial velocity alone. Perelmuter, Brodie \& Huchra (1995) circumvented this problem by comparing each candidate's color to the relative strengths of the calcium $\mathrm{K}$ and $\mathrm{H} \delta$ absorption lines in its spectrum. Unfortunately, the low throughput of LRIS below $4000 \AA$ and the noncoverage of that part of the spectrum for many of our candidates prevented us from doing likewise. However, since our field is within $5^{\prime}$ of the center of M81 where the cluster surface density is high, it is very likely that candidates whose spectra contain typical globular cluster features are members of the M81 globular cluster system. This assertion is supported by the fact that the Bahcall \& Soneira (1980) models predict fewer than 2 galactic stars in our $48 \operatorname{arcmin}^{2}$ field (the LRIS field is $\left.6^{\prime} \times 8^{\prime}\right)$ in the direction of M81 and in the color and magnitude range of our candidates.

Fifteen of the 16 candidates observed have spectra that are typical of globular clusters and we henceforth treat them as such. Only one, object 12, appears at first to be ruled out as a globular cluster on the basis of its spectrum (see Figure 2). The sharp upturn at the blue end of the spectrum and what appears to be broad-line Balmer emission are decidedly "un-globular". Still, there are genuine globular cluster features in the spectrum of this object, occurring at wavelengths that are consistent with it belonging to M81. Curiously, the photometric study of Perelmuter \& Racine (1995) cataloged a B $-\mathrm{V}$ value of 1.0 for this ob- ject, which is well within the normal color range for a globular cluster. The color of an object with a spectrum such as object 12 would certainly be much bluer, and likely would not have satisfied the Perelmuter \& Racine (1995) color criteria for inclusion in their catalogue of candidates. Thus we suspect that a transient phenomenon was occurring in the cluster at the time of our observation. Preliminary reductions of a spectrum of object 12 recently obtained with the Echellette Spectrograph and Imager (ESI) on the Keck II telescope has confirmed our suspicion. Object 12 no longer shows an upturn at the blue end of the spectrum and no broad-line emission features are present. Thus we consider object 12 to be a genuine M81 globular cluster and include it in our kinematic analysis of the M81 globular cluster system (Section 4). However, because the continuum shape is pivotal for accurate measurements of absorption line strengths and hence metallicity, we exclude object 12 from our chemical abundance and metallicity analysis in Section 3. The exact nature of the phenomenon occurring during the original observation of object 12 will be the subject of a forthcoming paper.

\subsection{Measurement of Radial Velocities}

A radial velocity was determined for each cluster by cross-correlating its non-flux-calibrated spectrum with three high signal-to-noise templates of M31 globular clusters. The actual crosscorrelation was accomplished using the IRAF routine $f x c o r$ in the $r v$ package. One of the templates (225-280) was obtained on the same run as these data and the other two (225-280 and 158-213) were obtained on a subsequent run, also with LRIS and at the same resolution. The ID numbers and heliocentric velocities of the template objects are from Huchra, Brodie \& Kent (1991). The template spectra have the same resolution as the cluster spectra, 5-6 , corresponding to a velocity resolution of roughly $300 \mathrm{~km} \mathrm{~s}^{-1}$. The results from the three templates agree quite well, the differences being a small $(\sim 5-10 \%)$ fraction of the velocity uncertainties returned by fxcor. The heliocentric line-of-sight velocities presented in Table 2 are the average of the results from the three templates. 


\section{Abundances and Metallicities}

\subsection{Metallicities}

We derived each cluster's mean metallicity by taking the error-weighted average of metallicities predicted by the strength of several absorption features.

The absorption line indices were measured as described in Brodie \& Huchra (1990), but our method for estimating the uncertainties was handled slightly differently because our background subtraction technique was different.

The extraction routines we used (see section 2.2) allow for the output of a "variance" spectrum, which is actually the standard deviation for each pixel in the extracted, background-subtracted spectrum, based on the quality of the polynomial fit to the background and the number of rows over which the spectrum was summed before extraction. We used this "variance" spectrum to estimate the photon uncertainty on the average flux per angstrom in a given bandpass by summing the "variance" pixels in quadrature over entire bandpass and then dividing by the bandpass width:

$$
\left\langle\sigma_{B P}\right\rangle=\frac{\left[\sum_{j=1}^{n} \sigma_{p i x}^{2}\right]^{\frac{1}{2}}}{\lambda_{2}-\lambda_{1}},
$$

where $\left\langle\sigma_{B P}\right\rangle$ is the photon uncertainty on the average flux per angstrom in a generic bandpass, $\sigma_{p i x}$ is the standard deviation in a given pixel (as given by the "variance" spectrum), $n$ is the number of pixels in the bandpass and $\lambda_{2}$ and $\lambda_{1}$ are the lower and upper wavelength limits of the bandpass, respectively.

As in Brodie \& Huchra (1990), we computed the uncertainty in the measured value of an index in a particular spectrum, $\sigma_{i}$, by summing in quadrature the photon uncertainties on the flux of each bandpass used to compute the index, including the absorption feature bandpass itself and the two continuum bandpasses:

$$
\sigma_{i}^{2}=\sigma_{C 1}^{2}+\sigma_{L}^{2}+\sigma_{C 2}^{2},
$$

$C 1$ and $C 2$ stand for the two continuum bandpasses and $L$ stands for the absorption line bandpass.
For each cluster, both the indices themselves and the photon errors on the indices were computed separately for each of our five integrations. The final index values are a weighted average of the indices derived from each integration using $\frac{1}{\sigma_{i}^{2}}$ as the weight. The final index photon errors, $\sigma_{I}$, are given by the reduced photon error:

$$
\sigma_{I}=\left(\frac{1}{\sum_{k=1}^{N} \sigma_{i}^{2}}\right)^{-\frac{1}{2}} .
$$

Table 3 gives the metallicity predicted by each spectral feature and the error-weighted mean metallicity of each cluster. The uncertainty in the metallicities predicted by a given individual index were derived by simply propagating the error in that index, $\sigma_{I}$, through the linear relationships between the index and metallicity derived in Brodie \& Huchra (1990). The uncertainty in the errorweighted mean metallicity for each cluster is, in a sense, an internal error since it is the standard of the mean. The individual Brodie \& Huchra index values may be found in Table 4. Two of the features used by Brodie \& Huchra (1990) to predict metallicity $(\mathrm{CNB}$ and Delta) require measurements below $3900 \AA$. Since, as mentioned in Section 2, the continuum shape of the spectra is uncertain for regions below $3900 \AA$ and above $6500 \AA$ we measured the index value and predicted metallicities for these two features (when the spectral coverage was sufficient) but excluded them from our derivation of the clusters' mean metallicities.

The metallicities of our sample clusters span a range of $-1.49<[\mathrm{Fe} / \mathrm{H}]<-0.21$ with an errorweighted mean of $\langle[\mathrm{Fe} / \mathrm{H}]\rangle=-0.91 \pm 0.09$ and a dispersion of $0.29 \pm 0.07$ dex. This range is narrower than that spanned by either the Milky Way or M31 globular cluster systems. This may be due to a bias in our observed sample. As shown in Figure 3 the colors of our sample clusters are biased toward the red end of the color distribution. The mean projected galactocentric radius of our sample is $2.6 \mathrm{kpc}$. If a cluster population associated with a metal-rich bulge or thick disk exists in M81, then our luminosity-selected sample taken from a $6^{\prime} \times 8^{\prime}$ region centered at $2.6 \mathrm{kpc}$ is likely dominated by such clusters, and the mean metallicity of our observed sample is not likely to accu- 
rately represent the mean metallicity of the entire M81 cluster system. Indeed, the mean projected galactocentric radius of the Perelmuter, Brodie \& Huchra (1995) sample is significantly larger (9.6 $\mathrm{kpc}$ ) and yields a rather lower mean metallicity value of $[\mathrm{Fe} / \mathrm{H}]=-1.48 \pm 0.19$ as measured from a composite spectrum. Brodie \& Huchra (1991) derived nearly the same result. To within the errors, this value is consistent with the peak of the metal-poor population in the metallicity distributions of the Milky Way and M31 cluster systems $(-1.5$ and -1.6 , respectively). The mean metallicity of the M81 Keck sample is somewhat lower than the mean of the metal-rich peak of M31 and Milky Way cluster metallicity distributions. With such a small sample size we cannot say whether the metal-rich M81 clusters, as a group, have a lower metallicity than their counterparts in the other systems or if we are simply seeing the inclusion of a few clusters which belong to a metal-poor halo but have small projected galactocentric radii.

The absence of clusters in our sample with $[\mathrm{Fe} / \mathrm{H}]$ greater than -0.2 is not really surprising, given the rarity of such clusters in the Milky Way and M31. Only 7 of the 150 M31 clusters studied by Huchra, Brodie \& Kent (1991) have metallicities higher than -0.2 , and just three of the 134 Milky Way clusters listed in the McMaster's catalog (Harris 1996) fit this criteria.

In an attempt to estimate the mean metallicity of the entire M81 globular cluster system we derived the arithmetic mean of all the M81 clusters for which spectroscopic metallicities have been established. An error-weighted mean would not be useful here because the metallicity errors for the Keck sample are much smaller than the errors in the other two studies and the clusters from the Keck sample would entirely dominate the result. In Table 3 of their paper, Perelmuter, Brodie \& Huchra (1995) indicate that three of the clusters in their study overlap with the Brodie \& Huchra (1991) sample. After comparing the coordinates and colors of objects in the two studies we have identified all of the objects from Brodie \& Huchra (1991) as candidates in the Perelmuter \& Racine (1995) catalogue (see Table 5). There are indeed three clusters which are present in both studies but one of them was misidentified by Perelmuter, Brodie \& Huchra (1995). Using the nomenclature of their Table 4, it is cluster Id50415 that should be matched with G19, not Is50401. At any rate, we adopt the error-weighted average of the metallicities derived in the two studies for these three clusters. The arithmetic mean metallicity of the resulting sample of 44 clusters is $\langle[\mathrm{Fe} / \mathrm{H}]\rangle=$ $-1.25 \pm 0.13$ where the error is the standard error of the mean. The mean value is entirely consistent with simply taking an arithmetic mean of metallicities of either the Milky Way $(-1.30 \pm 0.05$ dex $)$ or M31 $(-1.14 \pm 0.04$ dex $)$ globular cluster systems. The dispersion about the mean is $0.88 \pm 0.10$ dex, larger than for the other two systems $(\sim 0.55$ dex). This is likely due to the sizeable uncertainties in the metallicities from Perelmuter, Brodie \& Huchra (1995) and Brodie \& Huchra (1991) rather than an intrinsically larger spread in the metallicities of the M81 globular clusters.

\subsection{Internal Reddening in M81}

Using the B-V color-metallicity relation established for Milky Way globular clusters by Couture et al. (1990), we computed metallicities predicted by their $\mathrm{B}-\mathrm{V}$ colors for the clusters in the Keck sample. Object 12 was excluded from this analysis (see Section 2). Figure 4 shows the photometric metallicities plotted against the metallicities derived from their spectra as described in Section 3.1. For reference we also show Milky Way clusters for which foreground reddening is low $\left(\mathrm{E}_{(\mathrm{B}-\mathrm{V})}<0.4\right)$ and the line where clusters would lie if photometric and spectroscopic metallicities were in perfect agreement In the upper panel, the M81 cluster colors were corrected for foreground reddening only. Even after such a correction, the majority still lie above the line, i.e., their spectroscopic metallicities are lower than their colors would predict. An obvious explanation is that the light from the M81 clusters suffers internal reddening, which is likely to be variable across the disk of the galaxy. The range of color excess that would have to be applied to get all of the M81 clusters above the line to lie directly on it is $0.01<$ $\mathrm{E}_{(\mathrm{B}-\mathrm{V})}<0.24$. Given both the intrinsic scatter and non-linearity of the true color-metallicity relation, we cannot know the precise reddening for the individual clusters. However, the mean of the range quoted above $\left\langle\mathrm{E}_{(\mathrm{B}-\mathrm{V})}\right\rangle=0.12$ is likely a reasonable estimate of the average internal reddening in M81. This value is slightly lower than the value quoted by Perelmuter \& Racine (1995) but to within the 
errors they are consistent. The lower panel of Figure 4 shows the results of applying an additional $0.1 \mathrm{mag}$ correction to the cluster colors and recomputing the photometric metallicities.

To investigate the degree to which reddening could affect our derived cluster metallicities, we further de-reddened the spectra of the three clusters which required the largest corrections to bring their photometric and spectroscopic metallicities into agreement and remeasured their metallicities. We found that de-reddening the spectra systematically increases our derived mean metallicities, but the average change is only 0.05 dex, which is a fraction of the average statistical uncertainty of $\sim 0.20$ dex. The errors themselves also systematically increase, due to increased scatter in the metallicity values predicted by individual spectral features. This exercise demonstrates that spectroscopic metallicity determinations are far less vulnerable to errors introduced by internal reddening, a significant problem for the method of determining metallicities by photometry alone. Spectroscopy is especially advantageous when observing clusters in the disks of spiral galaxies where patchy dust can make the internal reddening highly variable.

\subsection{Individual Absorption Line Strengths}

Our spectra have a sufficiently high signal-tonoise ratio to compare the strengths of individual absorption features to those of Milky Way and M31 globular clusters. The complete suite of Lick/IDS indices (González 1993; Trager 1998) for the globular clusters in our M81 sample are presented in Table 6a and Table 6b. Figure 5 shows the strengths of G4300, NaD, TiO1, CN2, $\langle\mathrm{Fe}\rangle$ and $\mathrm{Mg} 2$ plotted against $[\mathrm{MgFe}]$ strength for M81, Milky Way and M31 globular clusters. González (1993) defines $\langle\mathrm{Fe}\rangle$ as the arithmetic mean of the Fe5335 and Fe5270 indices, and $[\mathrm{MgFe}]$ as $\sqrt{\langle\mathrm{Fe}\rangle \times \mathrm{Mgb}}$. The relations between these indices and the $[\mathrm{MgFe}]$ index for the M81 clusters in the Keck sample are generally the same as for Milky Way and M31 globular clusters. We had sufficient spectral coverage to measure the CN2 strengths for five M81 clusters, in which we see no evidence of a $\mathrm{CN}$ enhancement (see panel $d$ of Figure 5).

The Mg2 index appears to be slightly depressed in M81 clusters when compared to M31 and Milky
Way clusters with similar $[\mathrm{MgFe}]$ values (see panel $e$ of Figure 5). In Figure 6 the values of Fe5270, $\mathrm{Fe} 5335$ and $\mathrm{Fe} 5406$ are plotted against Mg1, Mg2 and Mgb for M81, M31 and Milky Way clusters. Mg2 appears depressed in M81's globular clusters, relative to Fe5335 but it is completely in line for the other two iron indices. The depression of Mg2 in Figure 5 can be better described as an excess of $\mathrm{Fe} 5335$, causing enhanced $[\mathrm{MgFe}]$ values. But is it a real effect? We do not think it is likely. The strengths of the Fe5335 and Fe5270 indices, whose average makes up $\langle\mathrm{Fe}\rangle$ and thus affect the value of $[\mathrm{MgFe}]$, can be influenced by contamination from other elements. Of all the Lick/IDS iron indices, only Fe5406 is a pure measure of iron (Trager 1998), and the Fe5406 index values of the M81 clusters are quite consistent with the Milky Way and M31 clusters for all three of the magnesium indices. The relative abundances of magnesium and iron in M81's globular clusters appear to be completely consistent with Milky Way and M31 globular clusters, at least at the upper end of the metallicity range.

\subsection{Ages}

In Figure 7 we plot the age-sensitive index $\mathrm{H} \beta$ against Mg2 for M81 globular clusters. For reference we also show these index values for individual Milky Way and M31 globular clusters, taken from the work of Trager (1998). Isochrones from the evolutionary-synthesis models of Worthey (1994) and from Fritze-v.Alvensleben \& Burkert (1995) are overplotted. Although there are significant differences between the models (the Worthey models give lower $\mathrm{H} \beta$ absorption values at a given age and metallicity, probably because these models do not include blue horizontalbranch stars in their population-synthesis scheme) it is clear that all but one of the M81 clusters in our sample are old, i.e., their ages are similar to those of Milky Way and M31 globular clusters. The exception is object 15 , whose $\mathrm{H} \beta$ index value is quite high when compared to the other clusters in the sample. Strong $\mathrm{H} \beta$ absorption in globular clusters is often interpreted as indicating low cluster metallicity, since the horizontal branches of metal-poor clusters are typically bluer than those of metal-rich clusters. However, the model tracks are isochrones, showing the behavior of the $\mathrm{H} \beta$ equivalent width for simple stellar 
populations of a single age, for a range of metallicities. The strong $\mathrm{H} \beta$ absorption in this cluster cannot be explained by metallicity alone. In addition, the color of this cluster is much bluer than would be expected on the basis of its mean metallicity. This object may in fact be a young (3 Gyr) globular cluster. Similar clusters have been observed in both M33 and M31 (Brodie \& Huchra, 1990; 1991).

\section{Cluster Kinematics}

Using the maximum likelihood method of Pryor \& Meylan (1993) we find a mean line-of-sight velocity for the Keck sample of $-105 \pm 32 \mathrm{~km} \mathrm{~s}^{-1}$ and a velocity dispersion of $123 \pm 23 \mathrm{~km} \mathrm{~s}^{-1}$. The mean velocity value differs significantly from both the heliocentric systemic velocity of M81 ( $-34 \pm$ $4 \mathrm{~km} \mathrm{~s}^{-1}$ ) and the median cluster radial velocity found by Perelmuter, Brodie \& Huchra (1995) of $-18 \pm 32 \mathrm{~km} \mathrm{~s}^{-1}$. We note, however, that the spatial extent of our sample is small and biased to one side of the galaxy (see Figure 1). The Keck sample shows no significant evidence for rotation, but the spatial bias could be obscuring its presence. To increase the sample's size and spatial extent, we include the velocities of 25 confirmed clusters from the study of Perelmuter, Brodie \& Huchra (1995) as well as the unpublished velocities of eight additional objects from Brodie \& Huchra (1991). As mentioned in Section 3.1, we have matched all eight of the Brodie \& Huchra objects with members of the Perelmuter \& Racine (1995) candidate list (see Table 5). Based on their colors and/or proper motions, two of them are likely foreground stars. The remaining six objects are confirmed M81 globular clusters, three of which were also observed by Perelmuter, Brodie \& Huchra (1995). For these three we have adopted the error-weighted mean of the velocities from the two studies. We combined the three data sets into a sample containing 44 M81 clusters, which we henceforth refer to as the "full sample".

The full sample has a mean heliocentric lineof-sight velocity of $-34 \pm 23 \mathrm{~km} \mathrm{~s}^{-1}$, in excellent agreement with M81's systemic velocity. The lineof-sight velocity dispersion of the full sample is $133 \pm 19 \mathrm{~km} \mathrm{~s}^{-1}$, generally consistent with the velocity dispersions of the globular cluster systems of the Milky Way ( $\sigma \sim 100$; Armandroff, 1989; Da-
Costa \& Armandroff, 1995) and M31 $(\sigma \sim 150$; Huchra, 1993; Huchra, Brodie \& Kent, 1991). The line-of-sight dispersion of M104's globular cluster system is significantly larger $(\sigma \sim 260$; Bridges et al. 1997), but that is not unusual given its higher luminosity.

\subsection{Rotation}

Figure 8 shows the positions of the M81 globular clusters marked with circles at the location of each cluster. The size and thickness of the circles are indicative of the magnitude and direction of the cluster radial velocities. Receding clusters (thick-lined circles) tend to be located in the northwest, approaching clusters (thin-lined circles) tend to lie more to the southeast. This suggests that rotation is present in the M81 globular cluster system in a sense that is consistent with the disk (Adler \& Westpfahl 1996). A more quantitative analysis is demonstrated in Figure 9. Panel $a$ shows the velocities (relative to the galaxy) of the full sample of clusters as a function of position angle with respect to the galaxy center. We used the maximum-likelihood method of Pryor \& Meylan (1993) to determine a rotation velocity and rotation axis for the sample. The analysis assumes that the system is rotating as a solid body. Data points taken from true solid-body rotators would follow the sinusoids in Figure 9 quite closely, although some scatter would necessarily be present, since clusters at the same position angle will have a variety of perpendicular distances from the axis of rotation. The rotation velocity quoted for each sub-sample in Figure 9, Figure 10 and in Table 7 is the linear speed of a point in the solid body which the sinusoid represents taken at the mean projected galactocentric radius of the sub-sample. For the full sample we find a rotation velocity of $85 \pm 32 \mathrm{~km} \mathrm{~s}^{-1}$ about an axis that is $17 \pm 28$ degrees east of north. This axis, shown by the arrow in Figure 8, is roughly aligned with the galaxy's minor axis and also the rotation axis for M81's gas (Adler \& Westpfahl 1996). For comparison, the same analysis of 182 M31 globular cluster velocities yields a rotation amplitude of $55 \pm 14 \mathrm{~km} \mathrm{~s}^{-1}$ and a line-of-sight dispersion of $138 \pm 8 \mathrm{~km} \mathrm{~s}^{-1}$. Here too, the rotation axis derived for the entire sample of M31 clusters is roughly aligned with M31's minor axis.

Given the division of the Milky Way and 
M31 globular cluster systems into kinematic subsystems which differ in their spatial and chemical characteristics, it is of interest to look for evidence of such sub-populations in the globular cluster system of M81. Since the spectroscopic metallicities of about two-thirds of the M81 full sample have large uncertainties, the clusters' $\mathrm{B}-\mathrm{R}$ colors must suffice for assigning them to one metallicity population or another. In the Milky Way globular clusters, the break between the metal-rich and metal-poor clusters corresponds to $\mathrm{B}-\mathrm{R} \sim 1.3$, so we adopt this value for separating the M81 clusters into red (metal-rich) and blue (metal-poor) sub-samples containing 33 and 11 members, respectively. We also examined a group of the 18 reddest clusters $(\mathrm{B}-\mathrm{R}>1.5)$. We realize that these sub-samples represent only rough metallicity groups and that, because of internal reddening (see Section 3.2), some clusters which belong in the low-metallicity group could be "scattered" into the high-metallicity group, i.e. the red subsamples may contain clusters that are not truly metal-rich.

The results of our kinematic analysis are summarized Table 7. Figure 9 illustrates that rotation is present in both red sub-samples at the $2 \sigma$ level, while the blue sub-sample shows no evidence for rotation. Additional analyses of sub-samples based on spatial characteristics alone showed that it is clusters at intermediate projected galactocentric radii that contribute most strongly to the global rotation signature (Figure 10). As Table 7 shows, the precise value of the rotation velocity for intermediate-range clusters varies depending on the choice of radius. We found the most significant detection in the $3-7 \mathrm{kpc}$ subsample $\left(157 \pm 37 \mathrm{~km} \mathrm{~s}^{-1}\right)$, but the other reasonable choices of radius limits $(2-6 \mathrm{kpc}$ and $4-8 \mathrm{kpc})$ yielded strong detections also. The presence of strong rotation in M81's globular clusters at intermediate projected galactocentric radii invites a comparison to the Milky Way thick-disk clusters noted by Armandroff (1989) which were further described by Burkert \& Smith (1997) as a "ring" of rapidly rotating metal-rich Milky Way clusters in a $2 \mathrm{kpc}$ wide annulus at intermediate galactocentric radius. Most of the M81 clusters in the intermediate-radius sample are members of the red, metal-rich sample, i.e., most have $\mathrm{B}-\mathrm{R}>1.3$. Unfortunately our sample is not large enough to make a direct comparison between metal-rich and metal-poor clusters in specific radius ranges, but at the very least our data are consistent with M81's metal-rich clusters at intermediate projected radii being associated with a thick disk in M81.

One may wonder why such a population was not detected in the globular cluster system of M31, given the much larger sample of clusters for which precise velocities and reliable spectroscopic metallicities have been measured. The main result of Huchra, Brodie \& Kent (1991)'s kinematic study was that the inner metal-rich clusters in M31 were the most rapidly rotating. The analysis that Ashman \& Bird (1993) performed on the Huchra, Brodie \& Kent (1991) data, showed strong statistical support for the division of M31's globular cluster system into disk and halo components. The method employed by Huchra, Brodie \& Kent (1991) was quite different than the one we use here. They used each cluster's projected distance along the galaxy major axis to bin the metal-rich and metal-poor clusters into spatial sub-samples. Then, for each metallicity group they simply computed the difference in the average heliocentric radial velocity for clusters on either side of the galaxy, lying beyond a given distance along the major axis. They estimated the rotation velocity to be one-half of this value. They found the difference between the metal-rich and metal-poor cluster rotation velocities to be most pronounced at very small distances along the galaxy major axis $(<1.5 \mathrm{kpc})$ with less pronounced differences persisting out to distances of roughly $8 \mathrm{kpc}$. They did not examine sub-samples in specific ranges of projected radii along the galaxy major axis, so their analysis did not address the question of whether the rotation of clusters at intermediate projected radii might show even stronger differences between the metal-rich and metal-poor groups.

We performed the maximum-likelihood kinematic analysis employed for the M81 clusters on 166 M31 clusters for which spectroscopic metallicities exist (Barmby et al. 2000). Table 8 shows that, as in the Milky Way, the most significant difference between the rotation of the metalrich and metal-poor clusters occurs at intermediate projected galactocentric radii. For all reasonable choices of radii, the difference between the metal-rich and metal-poor clusters is quite 
striking. Admittedly, treating the rotation axis as a free parameter greatly increases the chance for detecting rotation in a small sample. As a case in point, our analysis yields a weak detection of rotation with approximately the same amplitude but with very different rotation axes for metal-rich and metal-poor clusters lying inside 2 kpc. However, most of the metal-poor sub-samples have poorly-constrained rotation axes and have rotation-velocity-to-velocity-dispersion ratios that are consistent with their being supported primarily by thermal motion rather than by rotation. (The metal-rich M31 clusters inside $2 \mathrm{kpc}$ also have a low $\frac{v_{\text {rot }}}{\sigma_{l o s}}$, indicating that they may be associated with M31's bulge.) The metal-rich sub-samples all have their rotation axes roughly aligned with the galaxy's minor axis and they are rotating in the same sense as M31's gas (Loinard, Allen \& Lequeux 1995). Still, as a check on the extent to which treating the rotation axis as a free parameter could influence the value of the rotation velocity derived for the M31 sub-samples, we fixed the rotation axis to be aligned with M31's minor axis and repeated the analysis. To within the uncertainties, this procedure little or no change in the rotation velocities for all sub-samples except for the metal-poor clusters inside $2 \mathrm{kpc}$, which exhibit no rotation at all about the galaxy's minor axis. Thus the main result of Huchra, Brodie \& Kent (1991), who assumed the clusters were rotating about the galaxy minor axis, is recovered here. However, our analysis also reveals a potential thick-disk population among M31's metal-rich globular clusters, analogous to those found in the Milky Way and for which we see strong evidence in the globular clusters of M81.

While nature has not provided a sufficient number of metal-rich clusters residing at intermediate projected galactocentric radii to ever construct samples large enough to allay concerns about small-number statistics, the fact that all three spiral galaxies whose globular cluster systems have been studied in reasonable spectroscopic detail show evidence for similar populations offers encouragement that they are part of analogous stellar populations, which are possibly the thick disk components of these galaxies.

\subsection{Projected Mass}

The velocity distribution of the globular clusters can be used to give an estimate of the mass of the parent galaxy. To this end we follow the method of Heisler, Tremaine \& Bahcall (1985) and use the projected mass estimator

$$
M_{p}=\frac{f_{p}}{G(N-\alpha)}\left(\sum_{i}^{N} V_{i}^{2} r_{i}\right) .
$$

We adopt a value of $\alpha=1.5$, as recommended by Heisler, Tremaine \& Bahcall (1985) and assume a value of $f_{p}=\frac{32}{\pi}$. The latter corresponds to the case that the clusters are on isotropic orbits. If the clusters are on radial orbits, then the derived projected mass will be lower by a factor of 2. Based on the full sample of 44 clusters, the projected mass of M81 enclosed within $21.3 \mathrm{kpc}$ is $4.0 \pm 0.8 \times 10^{11} \mathrm{M}_{\odot}$. The error quoted is the dispersion in the distribution of projected masses computed from 1000 simulated data sets generated using the bootstrap method. This is only an approximation of the statistical error in the value of $\mathrm{M}_{\text {proj. }}$ It does not account for any error introduced by incorrect assumptions about the nature of the cluster orbits.

Tenjes, Haud \& Einasto (1998) quote a total luminosity for M81 of $\left(\mathrm{L}_{\mathrm{B}}\right)_{\mathrm{M} 81}=1.8 \pm 0.3 \times 10^{10} \mathrm{~L}_{\odot}$. Using the mass derived above we determine a lower limit for M81's mass-to-light ratio of $\left(\mathrm{M} / \mathrm{L}_{\mathrm{B}}\right)_{\mathrm{M} 81}>22$, in solar units.

Figure 11 shows a rough mass profile for M81 based on masses derived using clusters inside projected radii of $4,8,15$ and $22 \mathrm{kpc}$. The specific values are listed in Table 9 . The enclosed mass increases at large radius, indicative of a dark matter halo around M81. Evidence for a dark matter halo was also detected by Perelmuter, Brodie \& Huchra (1995).

\section{Discussion \& Conclusions}

We have obtained spectra of 16 globular cluster candidates in M81 with sufficient signal-tonoise to determine velocities and line-strengths for a number of absorption features. We also have supplemented our velocity data with velocities of 28 additional clusters from previous work, in order to investigate velocity structures. Our conclusions are as follows:

1. All 16 candidates we observed are confirmed as bona fide M81 globular clusters. One ap- 
pears to have been undergoing a transient event at the time of observation.

2. Our spectroscopic sample is biased to the metal-rich end of the metallicity range. Due to the spatial location of the clusters near the center of the galaxy, the Keck sample is likely to be dominated by bulge and/or thick-disk clusters. The former seems more likely, given the lack of a strong rotation signature in the Keck sample alone, but we note that rotation could be obscured by spatial bias in such a small sample. Based on the spectroscopically-determined metallicities of clusters in our sample, combined with metallicity estimates from previous work, we find the metallicity of the entire M81 globular cluster system to be similar to the metallicities of the Milky Way and M31 systems.

3. Nearly all the clusters' ages, based on $\mathrm{H} \beta$ absorption-line strengths, are consistent with ages of Milky Way and M31 clusters. However, one cluster in the sample may be as young as a few Gyrs. Similar young clusters have been observed in both M31 and M33.

4. Correlations between absorption line indices which have been established for globular clusters in the Milky Way and M31 hold for the M81 clusters as well, at least at the upper end of the metallicity-range where the Keck sample probes.

5. Based on the projected mass estimator, the mass of M81 is similar to the masses of the Milky Way and M31. The projected mass profile of M81 is consistent with the presence of a dark matter halo.

6. The M81 cluster system appears to be kinematically similar to the Milky Way and M31 systems in that the metal-rich clusters show evidence for significant rotation while the metal-poor clusters exhibit halo-like kinematics. The clusters which are rotating are doing so in the same sense as M81's disk and the global-rotation signature in our sample is driven primarily by clusters with projected galactocentric radii between 4 and $8 \mathrm{kpc}$. This may indicate the presence of a thick disk in M81. A similar analysis of the M31 globular cluster system suggests that such a population is present in that galaxy as well.

It is interesting to note that the annulus of rapidly-rotating, metal-rich clusters resides at the same galactocentric distance in all three galaxies discussed here. One interpretation is that the spatial and kinematic properties of these globular clusters do not obey galaxy scaling laws, but then the extent to which various components of the galaxies themselves follow scaling laws is not yet on firm footing. The thin (optical) disk of M31 is larger than that of the Milky Way (Walterbos \& Kennicutt 1988; Binney \& Tremaine 1987), and M31's metal-rich clusters extend farther than their counterparts in the Milky Way. Unfortunately the radial scale length of the thick disk component, with which the rotating metal-rich clusters are thought to be associated, is only partially constrained in the Milky Way (see Buser, Rong \& Karaali (1999) and references therein), and it has not been measured directly for either M31 or M81. Also, recent estimates of the dynamical masses of M31 and the Milky Way using satellite galaxies, distant globular clusters and planetary nebulae as tracers suggest that, in spite of its larger appearance, M31 may actually be less massive than the Milky Way (Evans \& Wilkinson 2000). Obviously more kinematic and metallicity data are needed for globular clusters in a variety of spirals to determine if an annulus of rapidly rotating metal-rich globular clusters are present in all spirals and, if not, whether its presence depends on any intrinsic or environmental property of the parent galaxy.

In summary, the globular cluster system of M81 is both kinematically and chemically very similar to the systems of the Milky Way and M31. This result lends support to the idea that universal processes govern the formation of globular cluster systems around spirals. It may also be relevant to the formation of early-type galaxies. As has been discussed extensively (Forbes, Brodie \& Grillmaire 1997; Ashman \& Zepf 1998), it is difficult to explain the globular cluster systems of high specific 
frequency ellipticals by a simple spiral-merger scenario, but this mechanism could be relevant in producing low specific frequency ellipticals. The blue sub-populations of globular clusters in such galaxies, having originated in the spiral galaxy progenitors, should be very similar.

\section{Acknowledgments}

We thank Karl Gebhardt for the use of his maximum-likelihood code and many related conversations. We are grateful to Pauline Barmby for providing an up-to-date catalogue of velocities and metallicities for M31 globular clusters. Also, we thank both Graeme Smith and Francois Schweizer for carefully reading the paper and making excellent suggestions. This work was supported by National Science Foundation grant number AST990732 and Faculty Research funds of the University of California, Santa Cruz.

\section{REFERENCES}

Adler, D., Westpfahl, D. 1996, AJ, 111, 735

Armandroff, T. 1989, AJ, 97, 375

Armandroff, T. \& Zinn, R. 1988, AJ, 96, 92

Ashman, K.M. \& Bird, C.M. 1993, AJ, 106, 2281

Ashman, K.M. \& Zepf, S.E. 1992, ApJ, 384, 50

Ashman, K.M. \& Zepf, S.E., Globular Cluster Systems, Cambridge University Press

Bahcall, J.N. \& Soneira, R.M. 1980, ApJS, 44, 73

Barmby, P., Huchra, J.P., Brodie, J.P., Forbes, D.A. \& Grillmair C.J. 2000, AJ, 119, 727

Battistini, P.; Bonoli, F.; Braccesi, A.; Federici, L.; Fusi-Pecci, F.; Marano, B.; Borngen, F. 1987, A\&AS, 67, 447

Battistini, P., Bonoli, F., Braccesi, A., Fusi Pecii, F. Malagnini, M. \& Marano, B. 1980 Astronomy and Astrophysics Supplement, 42, 357

Battistini, P.L., Bonoli, F., Casavecchia, M., Ciotti, L., Federici, L. \& Fusi-Pecci, F. 1993 A\&A, 272, 77

Binney, J. \& Tremaine, S. 1987, Galactic Dynamics, Princeton University Press, p. 17
Bridges, T.J., Ashman, K.M., Zepf, S.E., Carter, D., Hanes, D.A., Sharples, R.M. \& Kavelaars, J.J. 1997, MNRAS, 284, 376

Brodie, J.P. \& Huchra, J.P. 1990, ApJ, 362, 503

Brodie, J.P. \& Huchra, J.P. 1991, ApJ, 379, 157

Burkert, A. \& Smith, G.H. 1997, ApJ, 474, L15

Burstein, D., Faber, S.M., Gaskell, C.M. \& Krum, N. 1984, ApJ, 287, 586

Burstein, D. \& Heiles, C. 1984, ApJS, 54, 33

Buser, R., Jianxiang, R. \& Karaali, S. 1999, A\&A, 348,98

Chandar, R., Bianchi, L., Ford, H. \& Holland, C. 1999a, ApJS, 122431

Chandar, R., Bianchi, L., Ford, H. \& Holland, C. 1999b, ApJ, 517668

Chandar, R., Bianchi, L., Ford, H., Holland, C. \& Salasnich, B. 1999c, ApJ, 111794

Christian, C. \& Schommer, R. 1982, ApJS, 49, 405

Christian, C. \& Schommer, R. 1988, AJ, 95, 704

Cohen, J., Persson, S. \& Searle, L. 1984, ApJ, 281, 141

Côté, P. 1999, AJ, 118, 406

Couture, J., Harris, W.E. \& Allwright, J.W.B 1990, ApJS, 73, 67

Cowley, A.P. \& Burstein D. 1988, AJ, 95, 1071

Crampton, D., Cowley, A.P., Schade, D. \& Chayer, P. 1985, ApJ, 288, 494

Da Costa, G. \& Armandroff, T. 1995, AJ, 109, 2533

Elson, R. \& Walterbos, R. 1988, ApJ, 333, 594

Evans, N.W. \& Wilkinson, M.I. 2000, MNRAS, 316,929

Forbes, D.A., Brodie, J.P. \& Grillmaire, C.J. 1997, AJ, 113, 1652 
Freedman, W., Hughes, S.M., Madore, B.F. Mould, J.R., Lee, M., Stetson, P., Kennicutt, R.C., Turner, A., Ferrarese, L., Ford, H., Graham, J.A., Hill, R., Hoessel, J.G., Huchra, J., Illingworth, G.D. 1994, 1994, ApJ, 427, 628

Fritze-v.Alvensleben, U. \& Burkert, A. 1995, A\&A, 300, 58

Gonzales, J.J. 1993, PhD Thesis, University of California, Santa Cruz

Harris, W.E. 1996, AJ, 112, 1487

Heisler, J., Tremaine, S. \& Bahcall, J. 1985, ApJ, 422, L9

Hesser, J., Shawl, S. \& Meyer, J. 1986, PASP, 98, 403

Horne, K. 1986, PASP, 98, 609

Huchra, J.P., Brodie, J.P. \& Kent, S.M. 1991, ApJ, 370, 495

Huchra, J.P., Stauffer, J. \& Van Speybroeck, L. 1982, ApJ, 259L, 57

Kent, S.M., Huchra, J.P. \& Stauffer, J. 1989, AJ, 982080

Loinard, L., Allen, R.J. \& Lequeux, J. 1995, A\&A, 301,68

Minniti, D. 1995, AJ, 109, 1663

Oke, J.B. et al. 1995, PASP 107, 375

Perelmuter, J.-M., Brodie, J.P., Huchra, J.P. 1995, AJ, 110,620

Perelmuter, J.-M., Racine, R. 1995 AJ, 109, 1055

Ponder, J., Burstein, D., O’Connel, R., Rose, J., Frogel, J., Wu, C., Crenshaw, D.M., Rieke, M. \& Tripicco, M. 1998, AJ, 116, 2297

Pryor, C. \& Meylan, G. 1993, In Structure and Dynamics of Globular Clusters, ASP Conf. Ser. 50, ed. S.G. Djorgovski and G. Meylan, (San Francsico:ASP), 357

Reed, L.G., Harris, G.L.H. \& Harris, W.E. 1994, AJ, 107, 555

Sargent, W., Kowal, C. Hartwick \& F., van den Bergh, S. 1977, AJ, 82, 947
Schommer, R., Christian, C., Caldwell, N., Bothun, G. \& Huchra, J. 1991, AJ, 101, 873

Schweizer, F. 1986, Science, 231, 227

Sharov, A. \& Lyutyj, V. 1984, Soviet Astronomical Letters, 10, 273

Tenjes, P., Haud, U. \& Einasto, J. 1998, A\&A, 335,449

Trager, S. 1998, PhD Thesis, University of California, Santa Cruz

Walterbos, R. \& Kennicutt, R.C. 1988, A\&A, 198, 61

Worthey, G. 1994, ApJS, 95, 107

Zinn, R. 1985, A\&A, 152, 65

Zinn, R. 1993, In ASP Conf. Ser. 48, The Globular Cluster-Galaxy Connection, ed. G. Smith \& J. Brodie, (San Francisco:ASP), 38

This 2-column preprint was prepared with the AAS LATEX macros v5.0. 
TABLE 1

Observed Cluster Candidates

\begin{tabular}{|c|c|c|c|c|c|c|c|c|c|}
\hline $\begin{array}{l}\text { Slitlet } \\
(1)\end{array}$ & $\begin{array}{l}\text { ID } \\
(2)\end{array}$ & $\begin{array}{c}\alpha(\mathrm{J} 2000) \\
(3)\end{array}$ & $\begin{array}{c}\delta(\mathrm{J} 2000) \\
(4)\end{array}$ & $\begin{array}{c}\Delta \alpha \\
(\underset{(5)}{\operatorname{arcmin}})\end{array}$ & $\begin{array}{c}\Delta \delta \\
(6) \\
(6)\end{array}$ & $\begin{array}{c}\mathrm{R}_{\mathrm{P}} \\
(7) \\
(\operatorname{arcmin})\end{array}$ & $\begin{array}{l}\mathrm{V} \\
(8)\end{array}$ & $\mathrm{B}-\mathrm{V}$ & $\begin{array}{c}\mathrm{V}-\mathrm{R} \\
(10)\end{array}$ \\
\hline 1 & 50285 & $09: 55: 55.090$ & $69: 00: 56.14$ & 1.908 & -2.970 & 3.530 & 18.54 & 1.10 & 0.66 \\
\hline 2 & 50304 & $09: 55: 49.326$ & 69:01:15.29 & 1.391 & -2.651 & 2.994 & 18.97 & 1.02 & 0.60 \\
\hline 3 & 50359 & $09: 55: 37.461$ & 69:02:07.58 & 0.329 & -1.779 & 1.810 & 18.35 & 1.04 & 0.59 \\
\hline 4 & 50378 & $09: 55: 57.872$ & $69: 02: 23.07$ & 2.154 & -1.521 & 2.637 & 19.24 & 1.05 & 0.62 \\
\hline 5 & 50418 & $09: 55: 54.642$ & $69: 02: 52.57$ & 1.865 & -1.030 & 2.130 & 18.45 & 1.04 & $0.5 \overline{7}$ \\
\hline 6 & 50460 & $09: 55: 51.466$ & $69: 03: 23.60$ & 1.580 & -0.512 & 1.661 & 18.80 & 0.97 & 0.46 \\
\hline 7 & 50514 & $09: 55: 48.157$ & $69: 03: 52.03$ & 1.284 & -0.039 & 1.285 & 19.05 & 1.00 & 0.54 \\
\hline 8 & 50667 & $09: 55: 22.295$ & $69: 05: 19.16$ & -1.025 & 1.414 & 1.746 & 18.01 & 1.04 & 0.56 \\
\hline 9 & 50690 & $09: 55: 21.587$ & 69:05:31.98 & -1.088 & 1.627 & 1.958 & 18.76 & 0.98 & 0.48 \\
\hline 10 & 50738 & $09: 55: 30.277$ & 69:06:06.17 & -0.312 & 2.197 & 2.219 & 20.08 & 0.84 & 0.68 \\
\hline 11 & 50759 & 09:55:35.841 & $69: 06: 25.53$ & 0.184 & 2.520 & 2.526 & 18.59 & 0.82 & 0.40 \\
\hline 12 & 50782 & 095533.042 & $\begin{array}{llll}69 & 0639.88\end{array}$ & -0.066 & 2.759 & 2.760 & 18.70 & 1.00 & 0.52 \\
\hline 13 & 50787 & $09: 56: 05.569$ & $69: 06: 42.97$ & 2.834 & 2.810 & 3.991 & 19.12 & 0.87 & 0.57 \\
\hline 14 & 50834 & $09: 55: 25.403$ & $69: 07: 14.72$ & -0.747 & 3.340 & 3.422 & 19.03 & 0.92 & 0.51 \\
\hline 15 & 50867 & $09: 55: 51.995$ & $69: 07: 39.32$ & 1.622 & 3.749 & 4.085 & 19.83 & 0.75 & 0.30 \\
\hline 16 & 50889 & $09: 55: 40.194$ & $69: 07: 30.82$ & 0.571 & 3.608 & 3.653 & 18.73 & 0.99 & 0.62 \\
\hline M81 Center & $\cdots$ & 09:55:33.780 & 69:03:54.34 & 0.000 & 0.000 & 0.000 & $\cdots$ & $\cdots$ & $\cdots$ \\
\hline
\end{tabular}

Note.-IDs, coordinates, magnitudes and colors were obtained from Perelmuter \& Racine (1995).

${ }^{(1)}$ Slitlet number on LRIS mask.

${ }^{(2)}$ ID number.

${ }^{(3,4)}$ Equatorial coordinates, J2000.

${ }^{(5,6)}$ Position offsets from M81 center (arcminutes).

(7) Projected radius from M81 center (arcminutes).

(8) Apparent V magnitude.

${ }^{(9,10)}$ Colors, uncorrected for reddening. 
TABLE 2

Velocities for 16 M81 Globular Clusters

\begin{tabular}{ccr}
\hline \hline $\begin{array}{c}\text { Slitlet } \\
(1)\end{array}$ & $\begin{array}{c}\text { ID } \\
(2)\end{array}$ & \multicolumn{1}{c}{\begin{tabular}{c}
\multicolumn{1}{c}{ vlos $\left._{\text {los }}{ }^{-1}\right)$} \\
$(3)$
\end{tabular}} \\
\hline 1 & 50285 & $-199(33)$ \\
2 & 50304 & $19(27)$ \\
3 & 50359 & $-131(17)$ \\
4 & 50378 & $-318(31)$ \\
5 & 50418 & $-63(33)$ \\
6 & 50460 & $59(32)$ \\
7 & 50514 & $-335(28)$ \\
8 & 50667 & $-144(09)$ \\
9 & 50690 & $-261(27)$ \\
10 & 50738 & $22(39)$ \\
11 & 50759 & $11(24)$ \\
12 & 50782 & $-200(17)$ \\
13 & 50787 & $-87(49)$ \\
14 & 50834 & $69(16)$ \\
15 & 50867 & $-13(29)$ \\
16 & 50889 & $-100(25)$ \\
\hline
\end{tabular}

${ }^{(1)}$ Slitlet number on LRIS mask.

${ }^{(2)}$ ID number from Perelmuter \& Racine (1995).

${ }^{(3)}$ Heliocentric line-of-sight velocity (error in parenthesis). 
TABLE 3

Brodie \& Huchra Metallicity

\begin{tabular}{|c|c|c|c|c|c|c|c|c|c|}
\hline $\begin{array}{l}\text { Slit } \\
\text { (1) }\end{array}$ & $\begin{array}{r}\Delta \\
(2)\end{array}$ & $\begin{array}{l}\text { CNB } \\
(3)\end{array}$ & $\begin{array}{l}\text { CNR } \\
(4)\end{array}$ & $\begin{array}{l}\mathrm{G} \\
(5)\end{array}$ & $\begin{array}{c}\mathrm{MgH} \\
(6)\end{array}$ & $\begin{array}{l}\text { Mg2 } \\
(7)\end{array}$ & $\begin{array}{c}\text { Fe52 } \\
(8)\end{array}$ & $\begin{array}{l}\mathrm{NaI} \\
(9)\end{array}$ & $\begin{array}{c}\langle[\mathrm{Fe} / \mathrm{H}]\rangle \\
(10)\end{array}$ \\
\hline 1 & $\cdots$ & $\cdots$ & $\cdots$ & $\cdots$ & -1.655 & -1.194 & -0.658 & -0.352 & -1.207 \\
\hline & $\ldots$ & $\ldots$ & $\ldots$ & $\ldots$ & 0.073 & 0.038 & 0.105 & 0.064 & 0.369 \\
\hline 2 & $\ldots$ & $\ldots$ & $\ldots$ & $\ldots$ & -0.460 & -0.812 & -0.832 & -0.776 & -0.707 \\
\hline & $\ldots$ & $\ldots$ & $\ldots$ & & 0.102 & 0.054 & 0.148 & 0.094 & 0.167 \\
\hline 3 & $\ldots$ & $\ldots$ & $\ldots$ & 0.030 & -0.513 & -0.216 & -0.218 & -0.407 & -0.211 \\
\hline & $\ldots$ & $\ldots$ & $\ldots$ & 0.191 & 0.067 & 0.036 & 0.100 & 0.063 & 0.193 \\
\hline 4 & $\ldots$ & $\cdots$ & $\cdots$ & $\ldots$ & -0.470 & -0.311 & -0.505 & -0.887 & -0.407 \\
\hline & $\cdots$ & $\cdots$ & $\cdots$ & $\cdots$ & 0.122 & 0.065 & 0.177 & 0.111 & 0.088 \\
\hline 5 & $\cdots$ & $\cdots$ & $\cdots$ & $\cdots$ & -1.210 & -1.062 & -0.972 & -0.821 & -1.086 \\
\hline & & $\cdots$ & $\cdots$ & $\cdots$ & 0.072 & 0.038 & 0.108 & 0.067 & 0.091 \\
\hline 6 & $\cdots$ & $\cdots$ & $\ldots$ & $\cdots$ & -1.785 & -1.415 & -1.262 & -1.383 & -1.493 \\
\hline & & $\cdots$ & & $\ldots$ & 0.099 & 0.052 & 0.151 & 0.095 & 0.206 \\
\hline 7 & $\ldots$ & $\ldots$ & $\ldots$ & $\ldots$ & -1.057 & -0.971 & -0.794 & -1.151 & -0.955 \\
\hline & & & & & 0.125 & 0.066 & 0.187 & 0.116 & 0.098 \\
\hline 8 & 0.104 & -0.169 & -0.706 & -0.615 & -0.710 & -0.744 & -0.754 & -1.082 & -0.698 \\
\hline & 0.057 & 0.350 & 0.070 & 0.122 & 0.053 & 0.028 & 0.079 & 0.047 & 0.058 \\
\hline 9 & -0.233 & -0.717 & -1.125 & -1.108 & -1.254 & -1.375 & -1.029 & -1.488 & -1.212 \\
\hline & 0.133 & 1.062 & 0.127 & 0.213 & 0.092 & 0.048 & 0.136 & 0.082 & 0.133 \\
\hline 10 & $\ldots$ & $\ldots$ & -1.399 & -1.811 & -1.618 & -1.209 & -0.750 & -1.286 & -1.322 \\
\hline & $\ldots$ & $\cdots$ & 0.758 & 0.928 & 0.348 & 0.183 & 0.527 & 0.380 & 0.356 \\
\hline 11 & $\ldots$ & $\ldots$ & -2.222 & -0.558 & -1.691 & -1.193 & -1.193 & -1.745 & -1.114 \\
\hline & $\ldots$ & $\ldots$ & 0.092 & 0.175 & 0.069 & 0.036 & 0.104 & 0.070 & 0.409 \\
\hline 13 & $\cdots$ & $\ldots$ & $\ldots$ & $\ldots$ & -1.074 & -1.099 & -0.948 & -1.568 & -1.055 \\
\hline & & & & & 0.149 & 0.078 & 0.208 & 0.135 & 0.062 \\
\hline 14 & -0.210 & -0.315 & -1.140 & -1.028 & -1.179 & -1.171 & -1.023 & -1.385 & -1.107 \\
\hline & 0.244 & 2.468 & 0.142 & 0.239 & 0.096 & 0.051 & 0.145 & 0.094 & 0.074 \\
\hline 15 & $\ldots$ & $\ldots$ & $\ldots$ & -0.080 & -1.981 & -1.251 & -0.400 & -1.066 & -1.014 \\
\hline \multirow{3}{*}{16} & $\ldots$ & $\ldots$ & $\ldots$ & 0.468 & 0.173 & 0.092 & 0.270 & 0.191 & 0.713 \\
\hline & $\ldots$ & $\ldots$ & $\ldots$ & $\ldots$ & -0.687 & -0.630 & -0.739 & -0.804 & -0.674 \\
\hline & 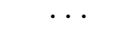 & $\ldots$ & $\cdots$ & $\cdots$ & 0.100 & 0.053 & 0.145 & 0.091 & 0.044 \\
\hline
\end{tabular}

Note. - There are two lines for each cluster. The first line contains the metallicity measurements. The second line contains the uncertainties in the metallicity measurements. The uncertainties for the individual metallicity measurements were determined by determining a "total error" for the index measurements, (equation 19 of Brodie \& Huchra, 1990) and then propagating that total error through their derived linear indexmetallicity relationships. The uncertainty quoted for the weighted mean metallicity for each cluster is weighted dispersion about weighted mean, where the weights were derived using equation 20 of Brodie \& Huchra, 1990.

(1) Slitlet number on LRIS mask.

${ }^{(2-9)}$ Metallicities predicted by various absorption line strengths, as described in Brodie \& Huchra (1990).

${ }^{(10)}$ Error-weighted mean, does not include $\Delta$ or NaI. 
TABLE 4

Indices Used to Predict Metallicity

\begin{tabular}{|c|c|c|c|c|c|c|c|c|}
\hline $\begin{array}{c}\text { Slitlet } \\
(1)\end{array}$ & $\begin{array}{l}\Delta \\
(2)\end{array}$ & $\begin{array}{c}\mathrm{CNB} \\
(3)\end{array}$ & $\begin{array}{c}\mathrm{CNR} \\
(4)\end{array}$ & $\begin{array}{l}\mathrm{G} \\
(5)\end{array}$ & $\begin{array}{c}\mathrm{MgH} \\
(6)\end{array}$ & $\begin{array}{c}\text { Mg2 } \\
(7)\end{array}$ & $\begin{array}{c}\text { Fe52 } \\
(8)\end{array}$ & $\begin{array}{r}\mathrm{NaI} \\
(9)\end{array}$ \\
\hline 1 & $\cdots$ & $\cdots$ & $\cdots$ & $\cdots$ & 0.009 & 0.103 & 0.070 & 0.139 \\
\hline & & & & & & & & 0.004 \\
\hline 2 & $\cdots$ & $\cdots$ & $\cdots$ & $\cdots$ & 0.067 & 0.141 & 0.062 & 0.110 \\
\hline 3 & $\ldots$ & ... & $\ldots$ & 0.218 & $\begin{array}{l}0.005 \\
0.064\end{array}$ & 0.005 & 0.007 & 0.007 \\
\hline & $\ldots$ & & & 0.017 & 0.003 & 0.004 & 0.005 & 0.004 \\
\hline 4 & $\cdots$ & $\cdots$ & $\cdots$ & $\cdots$ & 0.067 & 0.192 & 0.078 & 0.102 \\
\hline 5 & $\cdots$ & $\ldots$ & $\ldots$ & $\ldots$ & 0.006 & 0.007 & 0.009 & 0.008 \\
\hline & & & & & 0.004 & 0.004 & 0.005 & 0.005 \\
\hline 6 & $\cdots$ & $\cdots$ & ... & $\cdots$ & 0.003 & 0.080 & 0.040 & 0.068 \\
\hline & & & & & 0.005 & 0.005 & 0.007 & 0.007 \\
\hline$\gamma$ & $\cdots$ & $\cdots$ & $\cdots$ & $\cdots$ & 0.038 & 0.125 & 0.063 & 0.084 \\
\hline 8 & 0.796 & 0.269 & 0.066 & 0.161 & 0.055 & 0.148 & 0.065 & 0.088 \\
\hline & 0.018 & 0.053 & & 0.011 & 0.003 & 0.003 & 0.004 & 0.003 \\
\hline 9 & 0.690 & 0.186 & 0.009 & 0.118 & 0.028 & 0.084 & 0.052 & 0.060 \\
\hline & 0.042 & 0.162 & 0.017 & 0.019 & 0.004 & 0.005 & 0.007 & 0.006 \\
\hline 10 & $\ldots$ & $\ldots$ & $\begin{array}{r}-0.028 \\
0.103\end{array}$ & $\begin{array}{c}0.056 \\
0.081\end{array}$ & $\begin{array}{l}0.011 \\
0.017\end{array}$ & 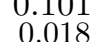 & $\begin{array}{l}0.066 \\
0.026\end{array}$ & $\begin{array}{l}0.074 \\
0.026\end{array}$ \\
\hline 11 & $\ldots$ & $\ldots$ & -0.140 & 0.166 & 0.007 & 0.103 & 0.044 & 0.043 \\
\hline 13 & $\ldots$ & $\ldots$ & & 0.015 & 0.003 & 0.004 & 0.056 & 0.0055 \\
\hline & & & & & 0.007 & 0.008 & 0.010 & 0.009 \\
\hline 14 & 0.698 & 0.247 & 0.007 & 0.125 & 0.032 & 0.105 & 0.052 & 0.067 \\
\hline+15 & 0.077 & 0.375 & 0.019 & 0.021 & 0.005 & 0.005 & & 0.006 \\
\hline 415 & & & & 0.208 & -0.007 & & & 0.090 \\
\hline t 16 & $\cdots$ & $\ldots$ & $\ldots$ & 0.041 & 0.008 & 0.009 & 0.066 & 0.0108 \\
\hline & & $\ldots$ & $\ldots$ & $\cdots$ & 0.005 & 0.005 & 0.007 & 0.006 \\
\hline
\end{tabular}

Note.-There are two lines for each cluster. The first line contains the index measurements. The second line contains the uncertainty (photon error) in the index measurements. Col.(1): Slitlet number on LRIS mask. Cols.(2)-(9): Absorption-line index values, measured as prescribed in Brodie \& Huchra 1990. Uncertainties measured as described in text. 
TABle 5

Data for Additional M81 Clusters

\begin{tabular}{|c|c|c|c|c|c|c|c|c|c|c|}
\hline $\begin{array}{l}\text { ID } \\
(1)\end{array}$ & $\begin{array}{c}\alpha(\mathrm{J} 2000) \\
(2)\end{array}$ & $\begin{array}{c}\delta(\mathrm{J} 2000) \\
(3)\end{array}$ & $\begin{array}{c}\Delta \alpha \\
\underset{(4)}{\operatorname{arcmin}})\end{array}$ & $\begin{array}{c}\Delta \delta \\
\left(\begin{array}{c}\operatorname{arcmin} \\
(5)\end{array}\right)\end{array}$ & $\begin{array}{c}R_{P} \\
(\operatorname{arcmin}) \\
(6)\end{array}$ & $\begin{array}{c}\mathrm{V} \\
(7)\end{array}$ & $\mathrm{B}-\mathrm{V}$ & $\mathrm{V}-\mathrm{R}$ & $\begin{array}{c}v_{l o s} \\
\left(\mathrm{~km} \mathrm{~s}^{-1}\right) \\
(10)\end{array}$ & $\begin{array}{c}{[\mathrm{Fe} / \mathrm{H}]} \\
(11)\end{array}$ \\
\hline $\mathrm{R} 9=\mathrm{HS} 18=50785^{* *}$ & $9: 54: 35.88$ & $69: 06: 43.1$ & -5.161 & 2.812 & 5.878 & 19.08 & 0.86 & 0.56 & $229(66)$ & $-1.00(0.84)$ \\
\hline $\mathrm{HS} 1=40312$ & $9: 54: 52.01$ & $69: 19: 45.3$ & -3.686 & 15.849 & 16.272 & 19.11 & 0.81 & 0.55 & $258(27)$ & $-2.10(0.97)$ \\
\hline $\mathrm{HS} 6=60072$ & $9: 55: 08.08$ & $68: 49: 56.5$ & -2.320 & -13.964 & 14.156 & 19.61 & 0.80 & 0.48 & $122(77)$ & $-1.77(0.83)$ \\
\hline $\mathrm{HS} 2=50864^{*}$ & $9: 56: 25.74$ & $69: 07: 27.8$ & 4.629 & 3.558 & 5.838 & 19.62 & 0.26 & 0.26 & $180(94)$ & $-2.09(0.59)$ \\
\hline $\mathrm{R} 12=60126^{*}$ & $9: 55: 26.97$ & $68: 44: 53.6$ & -0.617 & -19.013 & 19.023 & 19.16 & 0.99 & 0.59 & $-37(39)$ & $0.19(1.44)$ \\
\hline $\mathrm{R} 14=\mathrm{HS} 26=60012$ & $9: 55: 42.09$ & $68: 55: 00.6$ & 0.747 & -8.896 & 8.927 & 20.01 & 0.97 & 0.62 & $-129(57)$ & $0.40(2.65)$ \\
\hline $\mathrm{HS} 35=50960$ & $9: 55: 52.06$ & $69: 08: 18.7$ & 1.627 & 4.406 & 4.697 & 18.49 & 0.86 & 0.54 & $180(59)$ & $-1.43(0.40)$ \\
\hline $\mathrm{HS} 19=50415^{* *}$ & $9: 56: 20.58$ & 69:02:49.0 & 4.184 & -1.089 & 4.323 & 19.24 & 0.85 & 0.48 & $-218(51)$ & $-1.46(0.52)$ \\
\hline
\end{tabular}

*Likely to be a Galactic star, rejected from the sample.

**Also observed by Perelmuter et. al, 1995 (See Section 3.1.

${ }^{(1)}$ ID from Perelmuter \& Racine (1995).

${ }^{(2,3)}$ Equatorial Coordinates (J2000).

${ }^{(4,5)}$ Position offsets from M81 center (arcminutes)

${ }^{(6)}$ Projected radius from M81 center (arcminutes).

(7) Apparent V magnitude.

${ }^{(8,9)}$ Colors, uncorrected for reddening.

${ }^{(10)}$ Heliocentric, line-of-sight velocity (J. Huchra, private communication). Errors in parentheses.

${ }^{(11)}$ Metallicity from Brodie \& Huchra (1991). 
TABLE 6A

LICK IDS INDEX VALUES

\begin{tabular}{|c|c|c|c|c|c|c|c|c|c|c|c|c|}
\hline $\begin{array}{l}\text { Slit } \\
(1)\end{array}$ & $\begin{array}{c}\text { CN1 } \\
(2)\end{array}$ & $\begin{array}{c}\text { CN2 } \\
(3)\end{array}$ & $\begin{array}{c}\text { Ca4227 } \\
\text { (4) }\end{array}$ & $\begin{array}{c}\mathrm{G} 4300 \\
(5)\end{array}$ & $\begin{array}{c}\mathrm{Fe} 4383 \\
(6)\end{array}$ & $\begin{array}{c}\text { Ca4455 } \\
(7)\end{array}$ & $\begin{array}{c}\text { Fe4531 } \\
(8)\end{array}$ & $\begin{array}{c}\text { Fe4668 } \\
(9)\end{array}$ & $\begin{array}{l}\mathrm{H} \beta \\
(10)\end{array}$ & $\begin{array}{c}\text { Fe5015 } \\
(11)\end{array}$ & $\begin{array}{l}\text { Mg1 } \\
(12)\end{array}$ & $\begin{array}{l}\text { Mg2 } \\
(13)\end{array}$ \\
\hline 1 & $\cdots$ & $\cdots$ & $\cdots$ & $\cdots$ & $\ldots$ & $\cdots$ & $\cdots$ & $\cdots$ & 1.967 & 3.500 & 0.011 & 0.10 \\
\hline \multirow{3}{*}{2} & $\ldots$ & $\ldots$ & $\ldots$ & $\ldots$ & $\ldots$ & $\ldots$ & $\ldots$ & $\ldots$ & 0.197 & 0.414 & 0.004 & 0.004 \\
\hline & $\ldots$ & $\ldots$ & $\ldots$ & $\ldots$ & $\ldots$ & $\ldots$ & $\ldots$ & 0.790 & 1.903 & 3.688 & 0.068 & 0.139 \\
\hline & $\ldots$ & $\ldots$ & $\ldots$ & $\ldots$ & $\ldots$ & $\ldots$ & & 0.950 & 0.268 & 0.578 & 0.005 & 0.005 \\
\hline \multirow[t]{2}{*}{3} & $\ldots$ & $\ldots$ & $\ldots$ & 6.651 & 3.510 & 2.012 & 6.974 & 4.097 & 1.929 & 5.226 & 0.066 & 0.199 \\
\hline & $\cdots$ & $\cdots$ & $\cdots$ & 0.419 & 0.600 & 0.267 & 0.374 & 0.576 & 0.175 & 0.372 & 0.003 & 0.004 \\
\hline \multirow[t]{2}{*}{4} & $\cdots$ & $\cdots$ & $\cdots$ & $\ldots$ & $\ldots$ & $\cdots$ & $\ldots$ & $\ldots$ & 2.246 & 5.447 & 0.069 & 0.188 \\
\hline & $\cdots$ & $\cdots$ & & $\cdots$ & $\cdots$ & $\ldots$ & $\cdots$ & & 0.318 & 0.669 & 0.006 & 0.006 \\
\hline \multirow[t]{2}{*}{5} & $\cdots$ & $\cdots$ & $\cdots$ & $\cdots$ & $\cdots$ & $\cdots$ & $\cdots$ & $\cdots$ & 1.868 & 3.357 & 0.031 & 0.114 \\
\hline & $\cdots$ & $\cdots$ & & $\cdots$ & $\cdots$ & $\ldots$ & $\ldots$ & & 0.187 & 0.407 & 0.003 & 0.004 \\
\hline \multirow[t]{2}{*}{6} & $\ldots$ & $\ldots$ & $\cdots$ & $\ldots$ & $\ldots$ & $\ldots$ & $\ldots$ & 3.083 & 2.278 & 2.166 & 0.002 & 0.080 \\
\hline & $\cdots$ & $\ldots$ & $\ldots$ & $\ldots$ & $\ldots$ & $\ldots$ & & 0.852 & 0.249 & 0.563 & 0.005 & 0.005 \\
\hline \multirow[t]{2}{*}{7} & $\ldots$ & $\ldots$ & $\cdots$ & $\ldots$ & $\ldots$ & $\ldots$ & 4.119 & 3.279 & 1.832 & 2.684 & 0.040 & 0.123 \\
\hline & & $\ldots$ & & & & & 0.700 & 1.058 & 0.324 & 0.726 & 0.006 & 0.007 \\
\hline \multirow[t]{2}{*}{8} & 0.068 & 0.105 & 1.090 & 4.955 & 3.919 & 1.281 & 2.942 & 2.128 & 1.898 & 3.986 & 0.055 & 0.146 \\
\hline & 0.009 & 0.012 & 0.171 & 0.284 & 0.411 & 0.188 & 0.294 & 0.446 & 0.135 & 0.292 & 0.003 & 0.003 \\
\hline \multirow[t]{2}{*}{9} & 0.013 & 0.057 & 0.256 & 3.675 & 1.721 & 1.102 & 2.465 & 1.040 & 1.807 & 3.215 & 0.029 & 0.084 \\
\hline & 0.017 & 0.023 & 0.311 & 0.520 & 0.773 & 0.337 & 0.526 & 0.810 & 0.237 & 0.514 & 0.004 & 0.005 \\
\hline \multirow[t]{2}{*}{10} & -0.038 & -0.015 & 0.668 & 1.484 & 4.887 & 1.275 & 1.956 & 2.117 & $\begin{array}{l}1.769 \\
\end{array}$ & 6.273 & 0.010 & 0.100 \\
\hline & 0.102 & 0.127 & 1.623 & 2.420 & 2.811 & 1.262 & 1.973 & 2.949 & 0.896 & 1.842 & 0.017 & 0.018 \\
\hline \multirow[t]{2}{*}{11} & $-0.13 \overline{5}$ & -0.103 & 1.180 & 5.530 & 4.098 & 1.604 & 2.696 & 2.308 & 1.766 & 2.691 & 0.007 & 0.097 \\
\hline & 0.012 & 0.016 & 0.211 & 0.404 & 0.539 & 0.237 & 0.391 & 0.579 & 0.177 & 0.394 & 0.003 & 0.004 \\
\hline \multirow[t]{2}{*}{13} & $\ldots$ & $\ldots$ & & $\ldots$ & $\ldots$ & $\ldots$ & $\ldots$ & $\ldots$ & $\ldots$ & 2.946 & 0.036 & 0.112 \\
\hline & $\ddot{0} 0$ & $0 \ddot{035}$ & 0.544 & 3773 & $\begin{array}{l}394 \\
394\end{array}$ & $0 \ddot{834}$ & 2657 & 1540 & 2167 & 0.850 & $\begin{array}{l}0.007 \\
0.032\end{array}$ & $\begin{array}{l}0.008 \\
0.104\end{array}$ \\
\hline 14 & 0.019 & 0.026 & 0.343 & 0.582 & 0.850 & 0.363 & 0.552 & 0.845 & 0.250 & 0.547 & 0.005 & 0.005 \\
\hline \multirow[t]{2}{*}{15} & $\ldots$ & $\ldots$ & 1.178 & 6.072 & 7.390 & 1.291 & 4.693 & -0.874 & 4.509 & 6.897 & -0.005 & 0.099 \\
\hline & $\ldots$ & $\ldots$ & 0.649 & 1.050 & 1.457 & 0.711 & 1.051 & 1.544 & 0.407 & 0.888 & 0.008 & 0.009 \\
\hline \multirow[t]{2}{*}{16} & $\ldots$ & $\ldots$ & $\ldots$ & $\ldots$ & $\ldots$ & $\ldots$ & 3.681 & 5.688 & 1.643 & 4.092 & 0.057 & 0.157 \\
\hline & $\ldots$ & $\ldots$ & & $\cdots$ & $\cdots$ & $\ldots$ & 0.689 & 0.891 & 0.270 & 0.560 & 0.005 & 0.005 \\
\hline
\end{tabular}

(1) Slitlet number on LRIS mask.

${ }^{(2-13)}$ Absorbption-line index values measured as described in Trager 1998 and references therein. 
TABLE 6B

LiCK IDS InDEX VALUES, CONTINUED

\begin{tabular}{|c|c|c|c|c|c|c|c|c|c|c|c|c|c|}
\hline $\begin{array}{l}\text { Slit } \\
(1)\end{array}$ & $\begin{array}{l}\text { Mgb } \\
(14)\end{array}$ & $\begin{array}{c}\text { Fe5270 } \\
(15)\end{array}$ & $\begin{array}{c}\text { Fe5335 } \\
(16)\end{array}$ & $\begin{array}{c}\text { Fe5406 } \\
(17)\end{array}$ & $\begin{array}{c}\text { Fe5709 } \\
(18)\end{array}$ & $\begin{array}{c}\text { Fe5782 } \\
(19)\end{array}$ & $\begin{array}{l}\mathrm{NaD} \\
(20)\end{array}$ & $\begin{array}{l}\text { TiO1 } \\
(21)\end{array}$ & $\begin{array}{l}\text { TiO2 } \\
(22)\end{array}$ & $\begin{array}{l}\mathrm{H} \delta \mathrm{F} \\
(23)\end{array}$ & $\begin{array}{l}\mathrm{H} \gamma \mathrm{F} \\
(24)\end{array}$ & $\begin{array}{c}\mathrm{H} \delta \mathrm{A} \\
(25)\end{array}$ & $\begin{array}{c}\mathrm{H} \delta \mathrm{A} \\
(26)\end{array}$ \\
\hline 1 & 2.936 & 2.529 & 2.063 & 1.269 & 0.785 & 0.762 & 3.765 & 0.021 & 0.056 & $\cdots$ & $\cdots$ & $\cdots$ & $\ldots$ \\
\hline & 0.167 & 0.173 & 0.217 & 0.149 & 0.097 & 0.094 & 0.113 & 0.003 & 0.002 & $\ldots$ & $\ldots$ & $\ldots$ & $\ldots$ \\
\hline \multirow[t]{2}{*}{2} & 2.695 & 2.159 & 2.104 & 1.026 & 0.783 & 0.547 & 3.026 & 0.032 & 0.027 & $\ldots$ & $\ldots$ & $\ldots$ & $\ldots$ \\
\hline & 0.239 & 0.246 & 0.305 & 0.213 & 0.140 & 0.137 & 0.171 & 0.004 & 0.003 & $\ldots$ & & $\ldots$ & \\
\hline \multirow[t]{2}{*}{3} & 3.927 & 3.351 & 3.059 & 1.633 & 0.941 & 0.822 & 3.604 & 0.037 & 0.078 & $\ldots$ & -1.112 & $\ldots$ & -4.977 \\
\hline & 0.156 & 0.161 & 0.201 & 0.142 & 0.095 & 0.092 & 0.112 & 0.003 & 0.002 & $\ldots$ & 0.255 & $\ldots$ & 0.462 \\
\hline \multirow[t]{2}{*}{4} & 4.224 & 2.901 & 2.077 & 1.675 & 0.841 & 0.969 & 2.893 & 0.025 & 0.060 & $\ldots$ & $\ldots$ & $\ldots$ & \\
\hline & 0.274 & 0.289 & 0.356 & 0.245 & 0.167 & 0.160 & 0.203 & 0.005 & 0.004 & $\ldots$ & $\ldots$ & $\ldots$ & $\ldots$ \\
\hline \multirow[t]{2}{*}{5} & 2.444 & 1.903 & 1.858 & 0.754 & 0.640 & 0.682 & 2.933 & 0.019 & 0.028 & $\ldots$ & $\ldots$ & $\ldots$ & $\ldots$ \\
\hline & 0.171 & 0.180 & 0.226 & 0.159 & 0.10 & & 0.122 & 0.003 & 0.002 & $\ldots$ & $\ldots$ & $\ldots$ & $\ldots$ \\
\hline \multirow[t]{2}{*}{6} & 1.687 & 1.398 & 1.507 & 0.855 & 0.351 & 0.267 & 1.890 & 0.012 & 0.004 & $\ldots$ & $\ldots$ & $\ldots$ & $\ldots$ \\
\hline & 0.238 & 0.255 & 0.323 & 0.224 & 0.149 & 0.145 & 0.180 & 0.004 & 0.003 & $\ldots$ & $\ldots$ & $\ldots$ & $\ldots$ \\
\hline \multirow[t]{2}{*}{7} & 2.737 & 2.233 & 2.435 & 1.441 & 0.770 & 13 & 2.326 & 0.037 & 0.046 & $\ldots$ & $\ldots$ & $\ldots$ & $\ldots$ \\
\hline & 0.293 & 312 & 0.387 & 0.268 & 0.1 & & 0.215 & 0.005 & 0.004 & & & & \\
\hline \multirow[t]{2}{*}{8} & 2.635 & 37 & 2.081 & 33 & 0.7 & & 2.442 & 0.030 & $\ldots$ & 1.090 & -0.342 & -0.377 & -2.906 \\
\hline & 0.125 & 130 & 0.162 & 0.113 & 0.0 & & 0.087 & 0.002 & $\ldots$ & 0.242 & 0.164 & 0.381 & 0.288 \\
\hline \multirow[t]{2}{*}{9} & $\begin{array}{l}1.644 \\
\end{array}$ & 812 & 1.339 & 0.777 & 0.52 & & 1.689 & 0.012 & $\ldots$ & 1.854 & 1.177 & 0.730 & -0.410 \\
\hline & 0.219 & 0.228 & 0.294 & & 0.1 & & 0.156 & 0.004 & & 0.437 & 0.273 & 0.685 & 0.479 \\
\hline \multirow[t]{2}{*}{10} & 1.858 & 1.848 & 0.942 & 0.660 & 0.48 & & 2.012 & 0.046 & 0.025 & 0.274 & 1.891 & -0.310 & 0.700 \\
\hline & 0.840 & 0.883 & 1.174 & & & & 0.7 & & 0.013 & 2.700 & 1.155 & 5.514 & 2.054 \\
\hline \multirow[t]{2}{*}{11} & 3.463 & 1.720 & 0.905 & & 0.5 & & 1.103 & 0.011 & -0.014 & & -0.896 & & -3.318 \\
\hline & 0.156 & 0.174 & 0.226 & & 0.1 & & 0.135 & 0.003 & 0.003 & $\ldots$ & 0.232 & $\ldots$ & 0.409 \\
\hline \multirow[t]{2}{*}{13} & 2.486 & 031 & & & & & 1.528 & & 0.027 & $\ldots$ & $\ldots$ & $\ldots$ & \\
\hline & 0.348 & 0.346 & 0.432 & 0.31 & 0.197 & 0.1 & 0.257 & 0.005 & 0.005 & & & & \\
\hline \multirow[t]{2}{*}{14} & 1.498 & 79 & 1.534 & & 0.711 & & 1.715 & -0.005 & & 1.995 & 0.994 & 1.395 & 0.702 \\
\hline & 0.236 & 0.242 & 0.306 & 0.212 & 0.1 & 0.1 & 0.177 & 0.004 & & 0.483 & 0.307 & 0.749 & 0.525 \\
\hline \multirow[t]{2}{*}{15} & 0.696 & 2.455 & 2.548 & & -0.0 & & 2.264 & 0.0 & -0.041 & $\ldots$ & 1.400 & $\ldots$ & -5.866 \\
\hline & 0.448 & 0.4 & 0.554 & & & & 0.356 & & 0.007 & $\ldots$ & 0.626 & $\ldots$ & 1.273 \\
\hline \multirow[t]{2}{*}{16} & 3.358 & 2.321 & 2.077 & 1.569 & $0 . \overline{7}$ & & 3.027 & & 0.055 & $\ldots$ & & $\ldots$ & \\
\hline & 0.233 & 0.240 & 0.301 & 0.205 & 0.133 & 0.127 & 0.166 & 0.004 & 0.003 & $\ldots$ & $\ldots$ & $\ldots$ & \\
\hline
\end{tabular}

(1) Slitlet number on LRIS mask.

(14-26) Absorbption-line index values measured as described in Trager 1998 and references therein. 
TABLE 7

Kinematics of M81 Globular Clusters

\begin{tabular}{|c|c|c|c|c|c|c|c|}
\hline $\begin{array}{l}N \\
(1)\end{array}$ & $\mathrm{R}_{\mathrm{P}} \underset{(2)}{\text { Range }}$ & $\begin{array}{c}\left\langle\mathrm{R}_{\mathrm{P}}\right\rangle \\
(\mathrm{kpc}) \\
(3)\end{array}$ & $\begin{array}{c}\text { Color } \\
\text { Range } \\
(4)\end{array}$ & $\begin{array}{c}\left\langle v_{l o s}\right\rangle \\
\left(\mathrm{km} \mathrm{s}^{-1}\right) \\
(5)\end{array}$ & $\begin{array}{c}\sigma_{v_{l o s}} \\
\left(\mathrm{~km} \mathrm{~s}^{-1}\right) \\
(6)\end{array}$ & $\begin{array}{c}v_{\text {rot }} \\
\left(\mathrm{km} \mathrm{s}^{-1}\right) \\
(7)\end{array}$ & $\begin{array}{c}\theta_{\text {rot }} \\
\left(\begin{array}{c}\circ \\
(8)\end{array}\right)\end{array}$ \\
\hline 44 & Full Range & 7.9 & All & $-34(23)$ & $133(19)$ & $85(32)$ & $17(28)$ \\
\hline 33 & Full Range & 5.8 & $B-R>1.3$ & $-48(28)$ & $143(25)$ & $93(38)$ & $18(33)$ \\
\hline 11 & Full Range & 12.4 & $\mathrm{~B}-\mathrm{R}<1.3$ & $-11(15)$ & $11(48)$ & $21(26)$ & $143(52)$ \\
\hline 18 & Full Range & 5.2 & $\mathrm{~B}-\mathrm{R}>1.5$ & $-104(31)$ & $121(37)$ & $128(62)$ & $-11(39)$ \\
\hline 16 & $\mathrm{R}_{\mathrm{pgc}}<4 \mathrm{kpc}$ & 2.8 & All & $-103(31)$ & $135(42)$ & $89(84)$ & $-17(35)$ \\
\hline 22 & $2<\mathrm{R}_{\mathrm{pgc}}<6 \mathrm{kpc}$ & 3.6 & All & $-56(33)$ & $120(25)$ & $119(52)$ & $14(28)$ \\
\hline 17 & $3<\mathrm{R}_{\mathrm{pgc}}<7 \mathrm{kpc}$ & 4.2 & All & $-11(38)$ & $103(30)$ & $157(37)$ & $27(20)$ \\
\hline 13 & $4<\mathrm{R}_{\mathrm{pgc}}<8 \mathrm{kpc}$ & 5.3 & All & $1(46)$ & $109(37)$ & $168(42)$ & $45(25)$ \\
\hline 15 & $\mathrm{R}_{\mathrm{pgc}}>8 \mathrm{kpc}$ & 14.2 & All & $13(34)$ & $125(30)$ & $49(52)$ & $31(88)$ \\
\hline
\end{tabular}

(1) Number of clusters in sample.

${ }^{(2)}$ Spatial selection criteria for sample.

${ }^{(3)}$ Average $\mathrm{R}_{\mathrm{P}}$ of sample (assumes distance to M81 of $3.7 \mathrm{Mpc}$ ).

${ }^{(4)}$ Color selection criteria for sample.

${ }^{(5)}$ Mean heliocentric line-of-sight velocity of sample (errors in parentheses).

(6) Velocity dispersion, corrected for rotation (errors in parentheses).

(7) Amplitude of sample rotation (errors in parenthesis).

${ }^{(8)}$ Best fit position angle of rotation axis, positive angle is east of north. Errors in parentheses. 
TABle 8

Kinematics of M31 Globular Clusters

\begin{tabular}{|c|c|c|c|c|}
\hline $\begin{array}{l}\text { Spatial } \\
\text { Group } \\
(1)\end{array}$ & $\begin{array}{c}v_{\text {rot }} \\
\left(\mathrm{kms}^{-1}\right) \\
(2)\end{array}$ & $\begin{array}{c}\sigma_{v_{l o s}} \\
\left(\mathrm{~km} \mathrm{~s}^{-1}\right) \\
(3)\end{array}$ & $\begin{array}{c}\theta_{\text {rot }} \\
\left(\begin{array}{l}\circ \\
(4)\end{array}\right)\end{array}$ & $(5)^{\mathrm{N}}$ \\
\hline \multicolumn{5}{|l|}{$\overline{\mathrm{R}_{\mathrm{pgc}}<2 \mathrm{kpc}}$} \\
\hline MR & $96(56)$ & $152(36)$ & $109(56)$ & 17 \\
\hline MP & $81(60)$ & $153(68)$ & $-113(51)$ & 15 \\
\hline \multicolumn{5}{|l|}{$2<\mathrm{R}_{\mathrm{pgc}}<6 \mathrm{kpc}$} \\
\hline MR & $127(40)$ & $122(40)$ & $119(24)$ & 16 \\
\hline MP & $20(28)$ & $134(15)$ & $164(108)$ & 45 \\
\hline \multicolumn{5}{|l|}{$3<\mathrm{R}_{\mathrm{P}}<7 \mathrm{kpc}$} \\
\hline MR & $143(48)$ & $119(43)$ & $128(22)$ & 14 \\
\hline $\mathrm{MP}$ & $37(33)$ & $144(16)$ & $136(54)$ & 43 \\
\hline \multicolumn{5}{|l|}{$4<\mathrm{R}_{\mathrm{P}}<8 \mathrm{kpc}$} \\
\hline MR & $144(68)$ & $129(50)$ & $87(39)$ & 11 \\
\hline MP & $65(34)$ & $139(21)$ & $121(31)$ & 39 \\
\hline \multicolumn{5}{|l|}{$\mathrm{R}_{\mathrm{P}}<8 \mathrm{kpc}$} \\
\hline MR & $49(39)$ & $56(27)$ & $96(44)$ & 12 \\
\hline MP & $72(26)$ & 114 (13) & $98(29)$ & 36 \\
\hline
\end{tabular}

${ }^{(1)}$ Limits of annulus defining sample. Assumes distance of 744 kpc to M31.

${ }^{(2)}$ Best fit rotation velocity, errors in parentheses

${ }^{(3)}$ Velocity dispersion (corrected for rotation), errors in parentheses.

${ }^{(4)}$ Best fit rotation axis (positive angle is east of north), errors in parenthesis

${ }^{(5)}$ Number of clusters in sample.

Note. $-\mathrm{MR}=$ Metal-rich clusters, those with $[\mathrm{Fe} / \mathrm{H}]>=$ -0.8. MP indicates clusters with metallicity less than this value. 
TABle 9

Projected Mass of M81

\begin{tabular}{|c|c|c|}
\hline $\begin{array}{c}\mathrm{N} \\
(1)\end{array}$ & $\begin{array}{c}R_{\max } \\
(\mathrm{kpc}) \\
(2)\end{array}$ & $\begin{array}{c}\mathrm{M}_{\text {proj }} \\
\left(10^{11} \mathrm{M}_{\odot}\right) \\
(3)\end{array}$ \\
\hline 15 & 3.9 & $1.2(0.8)$ \\
\hline 30 & 7.8 & $2.4(0.7)$ \\
\hline 38 & 14.7 & $3.1(0.7)$ \\
\hline 46 & 21.3 & $4.0(0.8)$ \\
\hline
\end{tabular}

${ }^{(1)}$ Number of points in sample.

${ }^{(2)}$ Maximum projected galactocentric radius in sample.

${ }^{(3)}$ Projected mass interior to $\mathrm{R}_{\max }$.

${ }^{(4)}$ Statistical uncertainty in projected mass. Error (in parentheses) does not include errors potentially introduced by assumptions about the cluster orbits. 


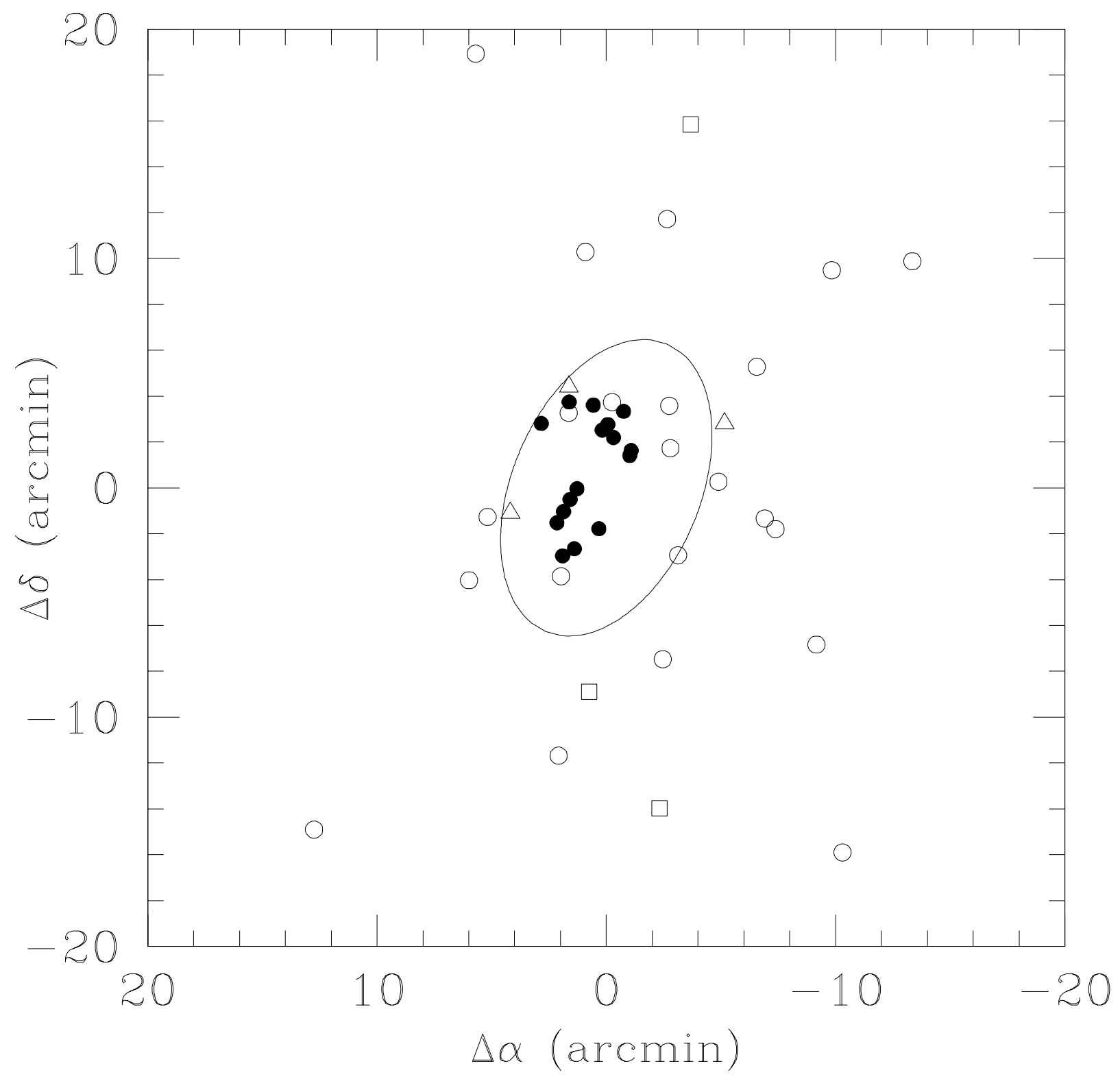

Fig. 1.- Positions of all spectroscopically-confirmed M81 globular clusters, relative to the center of M81. The ellipse shows the location and orientation of M81's disk, taken from Perelmuter, Brodie \& Huchra (1995) Solid circles are members of the Keck sample. Open circles are clusters studied by Perelmuter, Brodie \& Huchra (1995), and open squares are clusters from Brodie \& Huchra (1991) for which velocities have been measured (J. Huchra, private communication.) Open triangles indicate clusters studied by both Brodie \& Huchra (1991) and Perelmuter, Brodie \& Huchra (1995). 

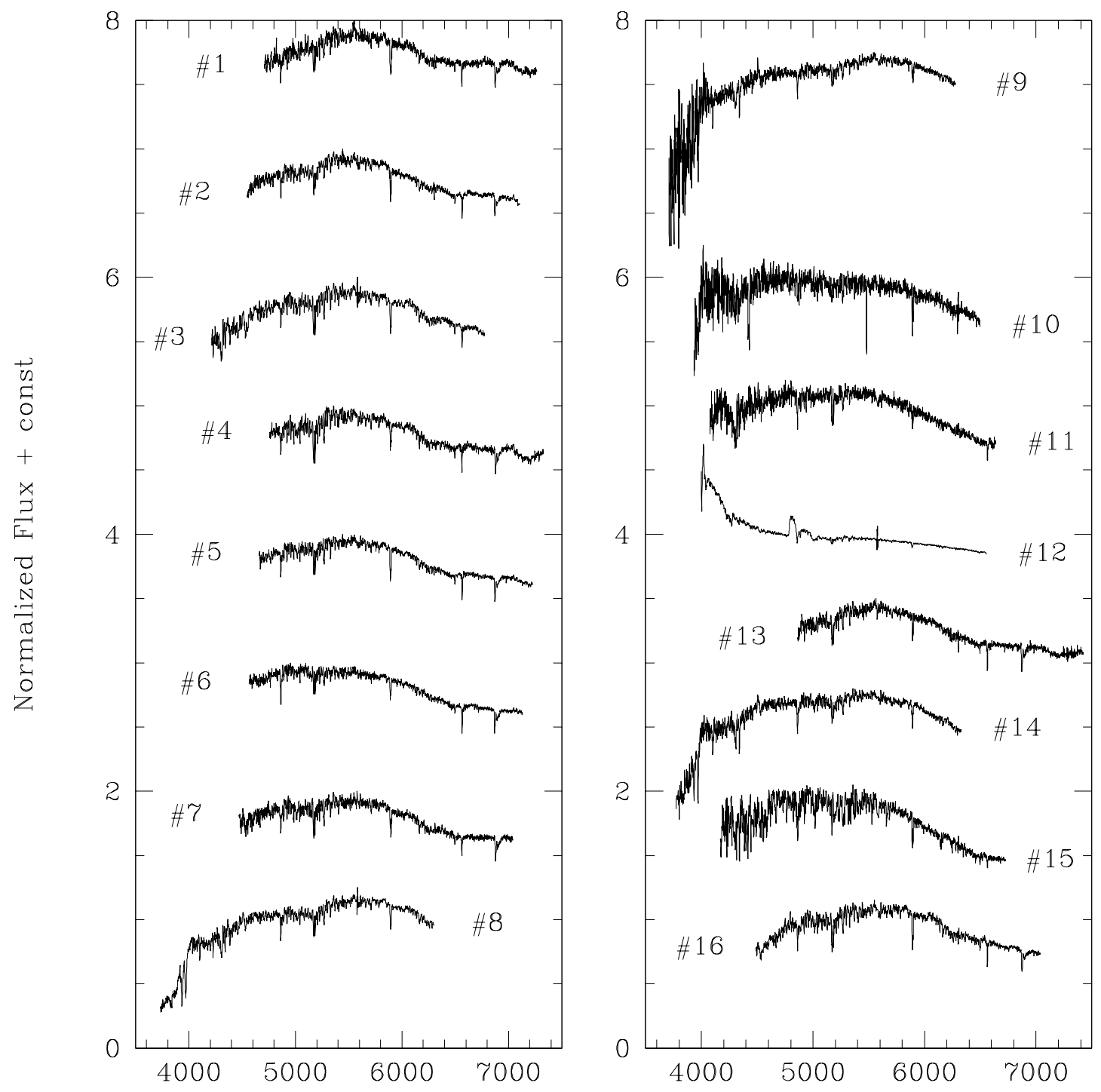

Wavelength $(\AA)$

Fig. 2.- Spectra of 16 M81 globular cluster candidates, obtained with 4500 seconds of integration with LRIS on the Keck I telescope. The spectra have been corrected for foreground reddening and shifted to the rest frame. Continuum shapes are reliable only between $3900 \AA$ and $6500 \AA$. All are confirmed as M81 globular clusters, although object 12 appears to have been observed during a transient event in the cluster (see Section 2). 

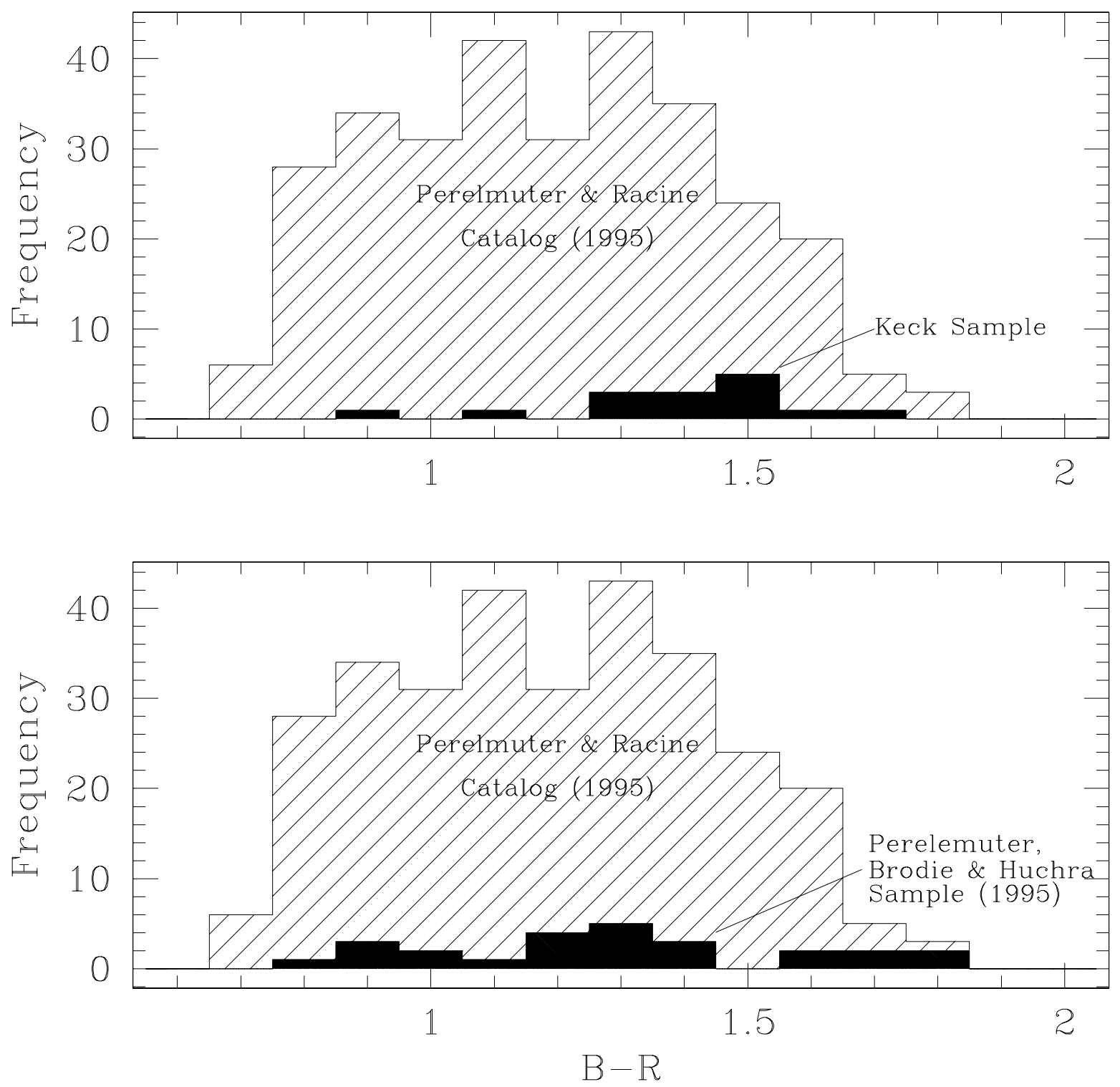

Fig. 3.- The B $-\mathrm{R}$ color distribution of the Keck sample (upper panel) and the sample of Perelmuter, Brodie \& Huchra (1995) (lower panel) compared to the color distribution of the entire list of M81 cluster candidates from Perelmuter \& Racine (1995). The Keck sample is clearly biased to the redder, metal-rich end of the spectrum. The Perelmuter, Brodie \& Huchra (1995) sample has a more uniform color distribution (see Section 3.2). 

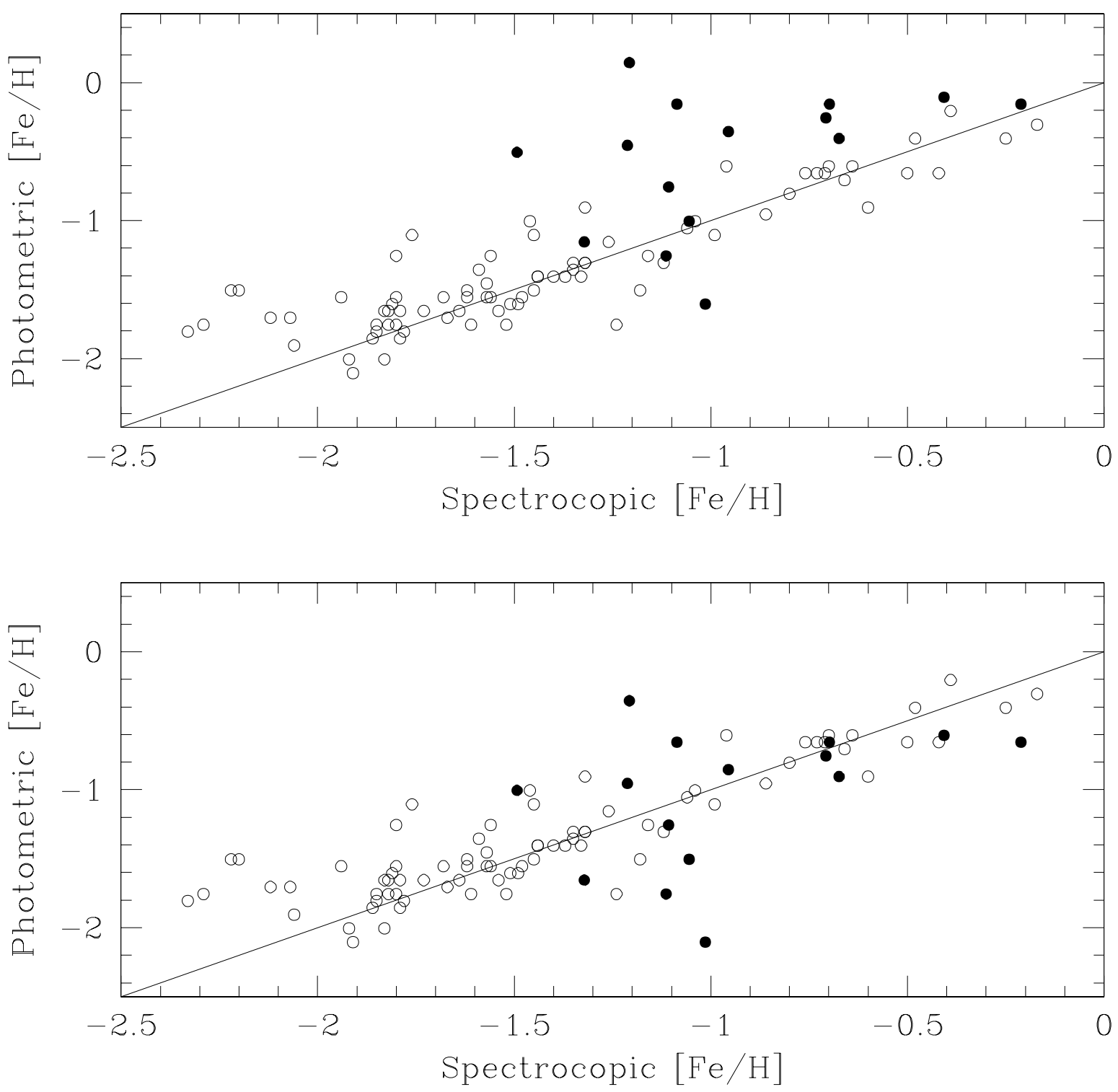

Fig. 4.- Photometric vs. spectroscopic metallicities for M81 (filled circles) and Milky Way (open circles) globular clusters. Photometric metallicities were computed from $\mathrm{B}-\mathrm{V}$ colors using the color-metallicity derived in Couture et al. (1990). In the upper panel, the colors were corrected for foreground reddening only, which was estimated to be $\sim 0.1$ (Perelmuter \& Racine 1995). The lower panel shows that de-reddening the M81 cluster colors by an additional 0.1 mag brings the photometric metallicities of the M81 clusters in line with those of the Milky Way (but see Section 3.2). Only Milky Way clusters for which foreground reddening is low $\left(\mathrm{E}_{(\mathrm{B}-\mathrm{V})}<0.4\right)$ are included. The reddening and metallicity values for the Milky Way clusters were obtained from Harris (1996). 

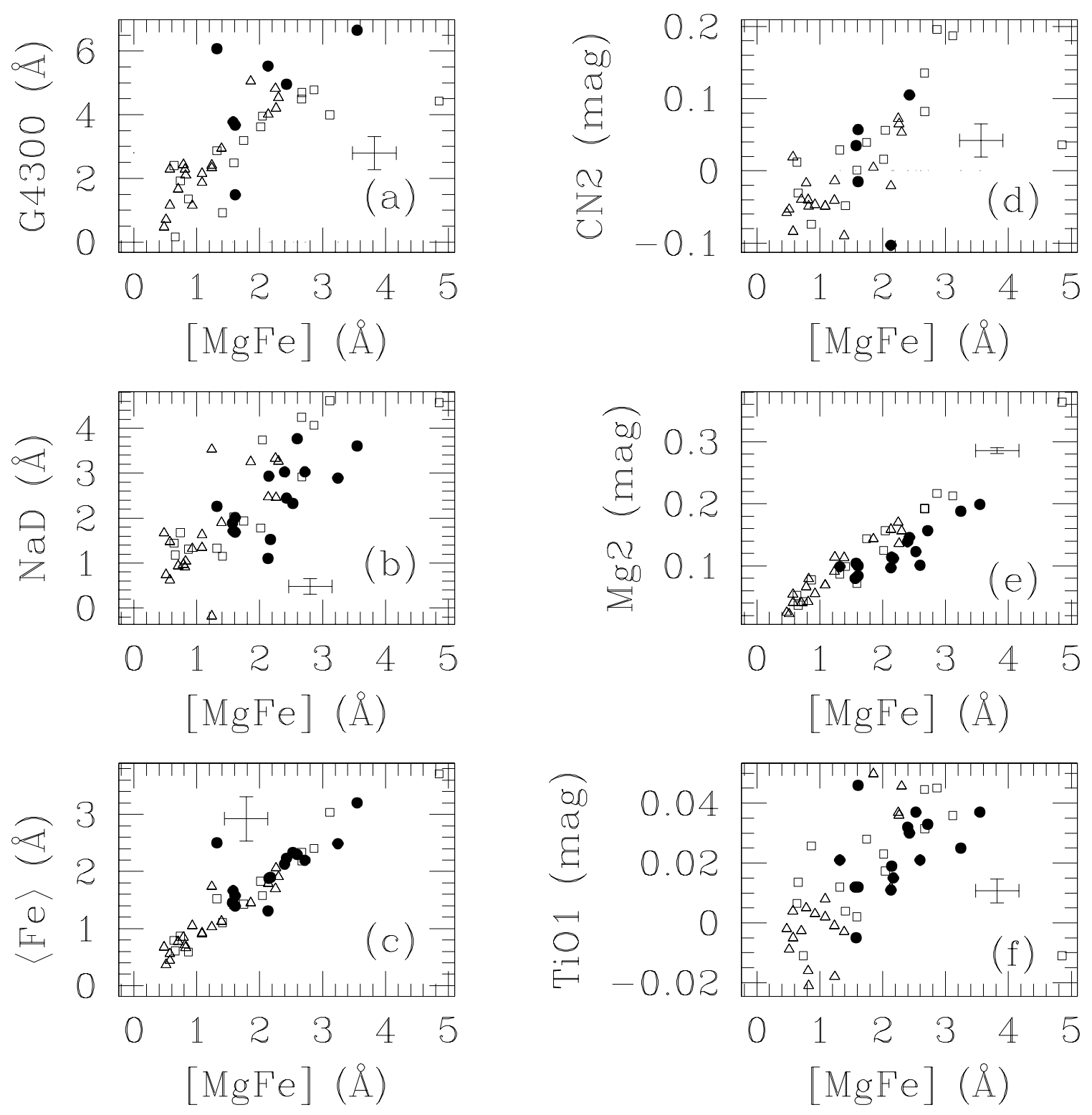

Fig. 5.- Selected Lick IDS indices plotted against the metallicity-sensitive index [MgFe] for M81 (filled circles), M31 (open squares) and Milky Way (open triangles) globular clusters. The error bars show the typical sizes of the statistical (photon) errors on the M81 cluster indices. The correlations between specific indices observed for Milky Way and M31 globular clusters are also present in the M81 globular cluster system. The apparent depression of $\mathrm{Mg} 2$ with respect to [MgFe] (panel $e$ ) is likely due to contamination of the Fe5335 index. See Section 3.1 and Figure 6 for more detail. 

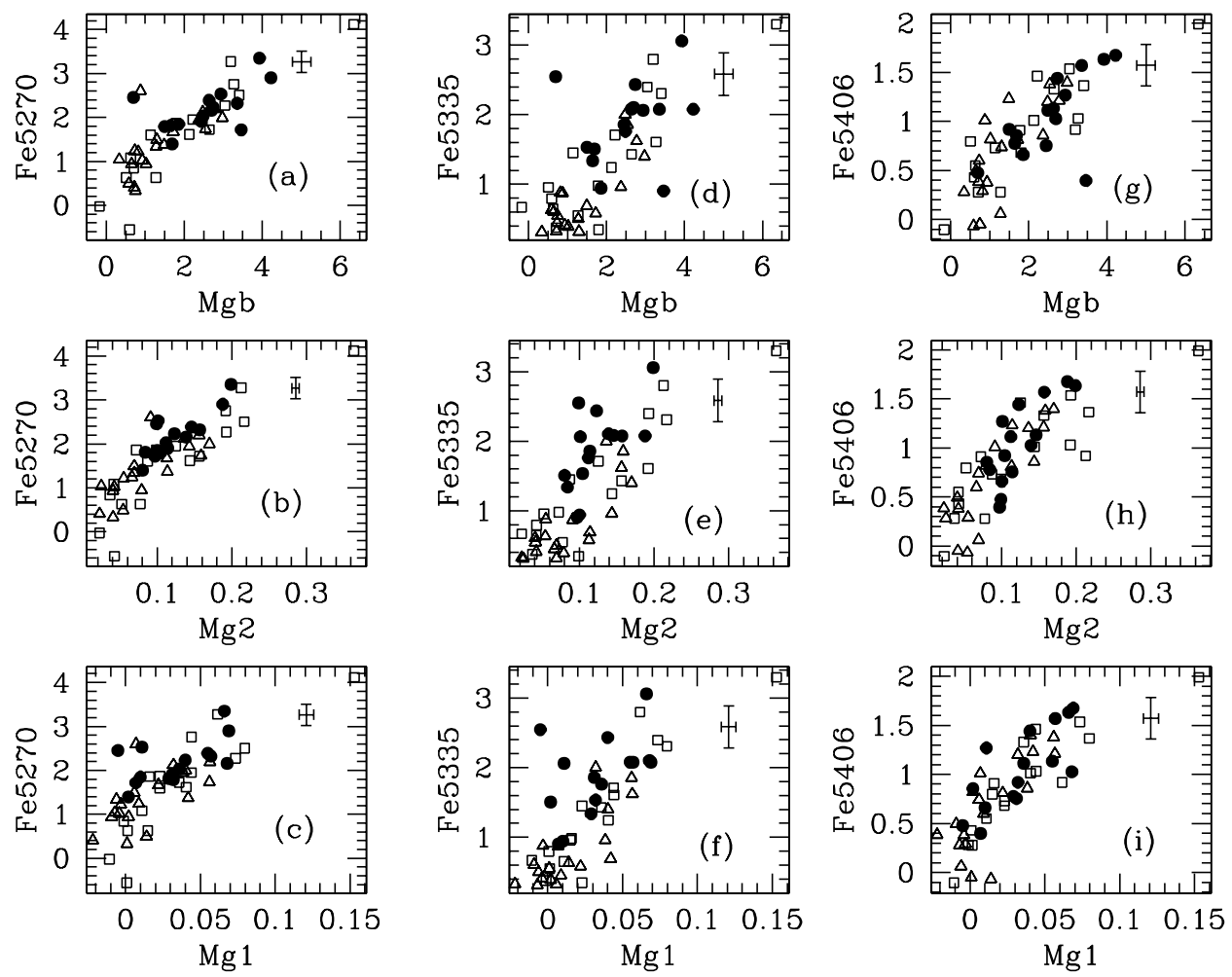

Fig. 6.- Iron indices plotted against magnesium indices for M81 (filled circles), M31 (open squares) and Milky Way (open triangles) globular clusters. The error bars show the typical sizes of the statistical errors for the M81 data. The Fe5335 index appears enhanced, but both Fe5335 and Fe5270 can be affected by the strength of absorption by other elements (Trager 1998). Fe5406 is a pure measure of the iron abundance, and based on this index we conclude that relative abundances of magnesium and iron in M81 globular clusters at the metal-rich end of the metallicity range is consistent with the abundances for globular clusters in M31 and the Milky Way. The apparent depression of Mg2 in panel $e$ of Figure 5 is likely due to contamination artificially enhancing the Fe5335 index. 


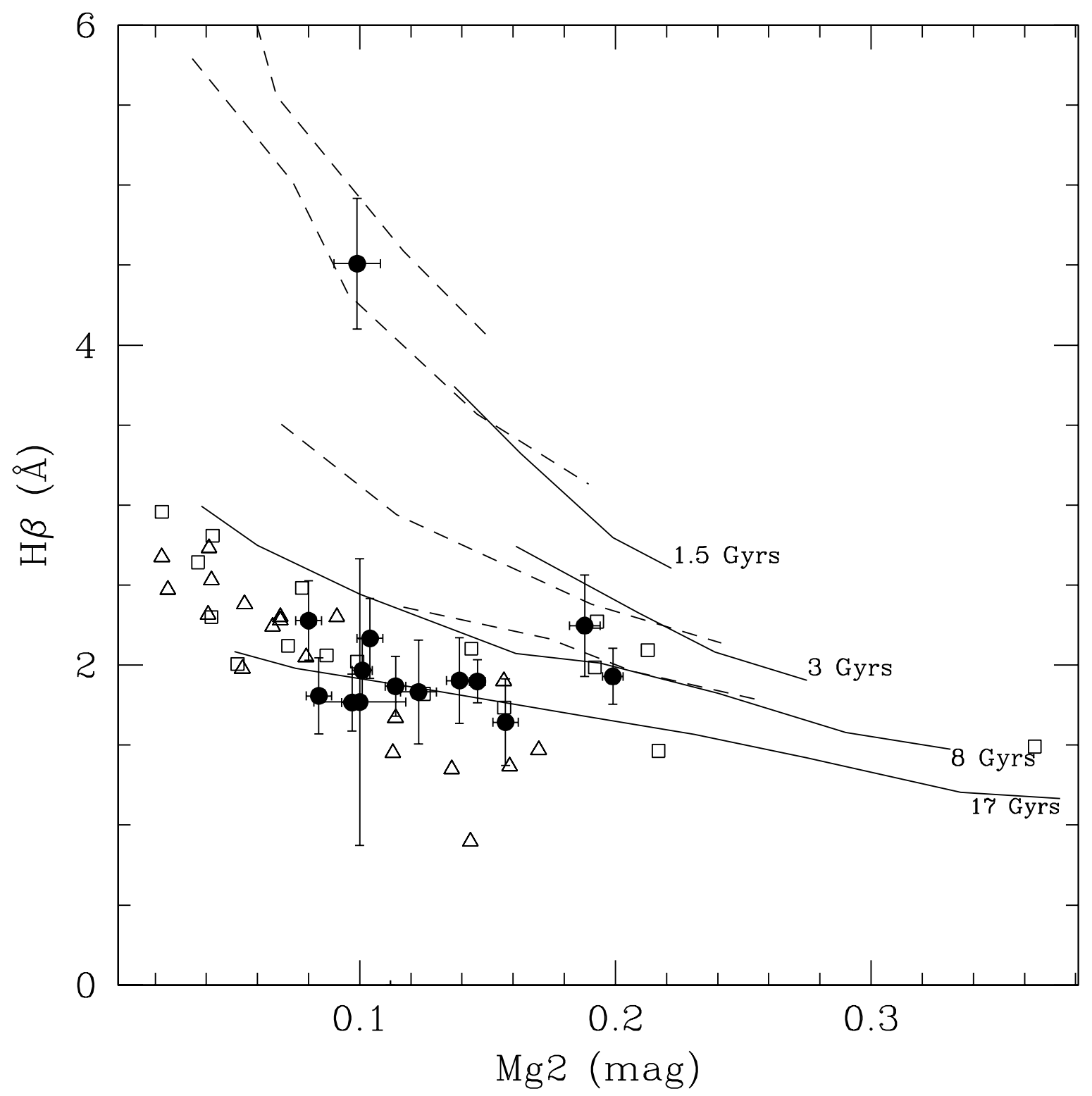

Fig. 7.- The age-sensitive index $\mathrm{H} \beta$ plotted against the metallicity-sensitive index Mg2 for M81 (filled circles), M31 (open squares) and Milky Way (open triangles) globular clusters. Overplotted as solid lines are Worthey (1994) isochrones of ages 1.5 and 3 Gyrs (spanning $[\mathrm{Fe} / \mathrm{H}]-0.225$ to 0.5 ) and 8 and 17 Gyrs (spanning $[\mathrm{Fe} / \mathrm{H}]$ of -2.0 to 0.5 ). The dashed lines are isochrones of ages $1.5,3,8$ and 15 Gyrs from the models of Fritze-v.Alvensleben \& Burkert (1995), spanning metal-content of $[\mathrm{Fe} / \mathrm{H}]=-1.3$ to solar. With the exception of object 15, the M81 cluster ages are consistent with the ages of M31 and Milky Way globular clusters, i.e., they are old. 


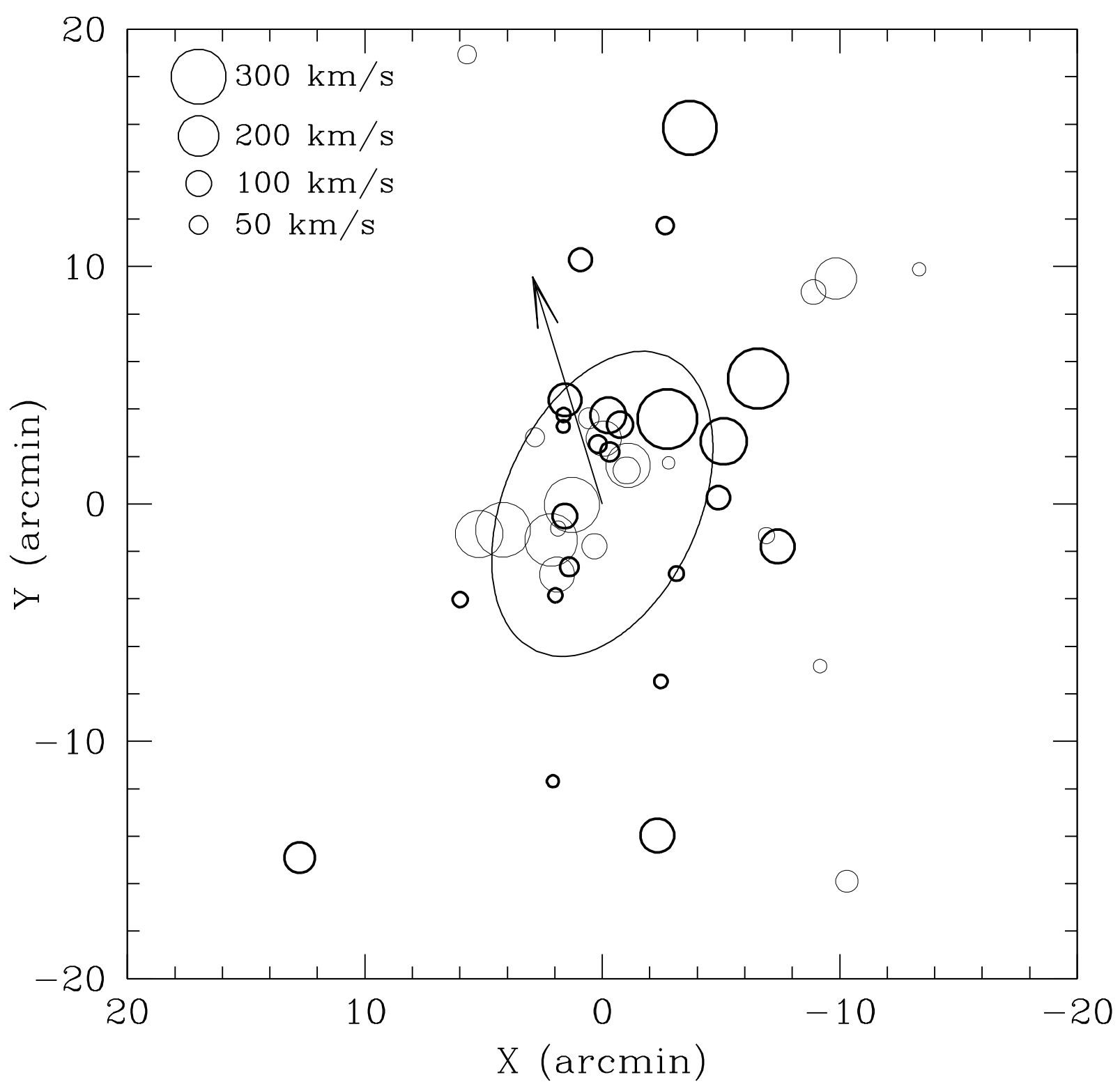

Fig. 8. - Velocities and positions of the full sample of M81 globular clusters. Thick-lined circles are receding, thin-lined circles are approaching. The ellipse shows the location of the M81's disk, taken from Perelmuter, Brodie \& Huchra (1995). The qualitative impression is that the M81 cluster system is rotating. The arrow shows the rotation axis for the full sample (see Section 4.1). 

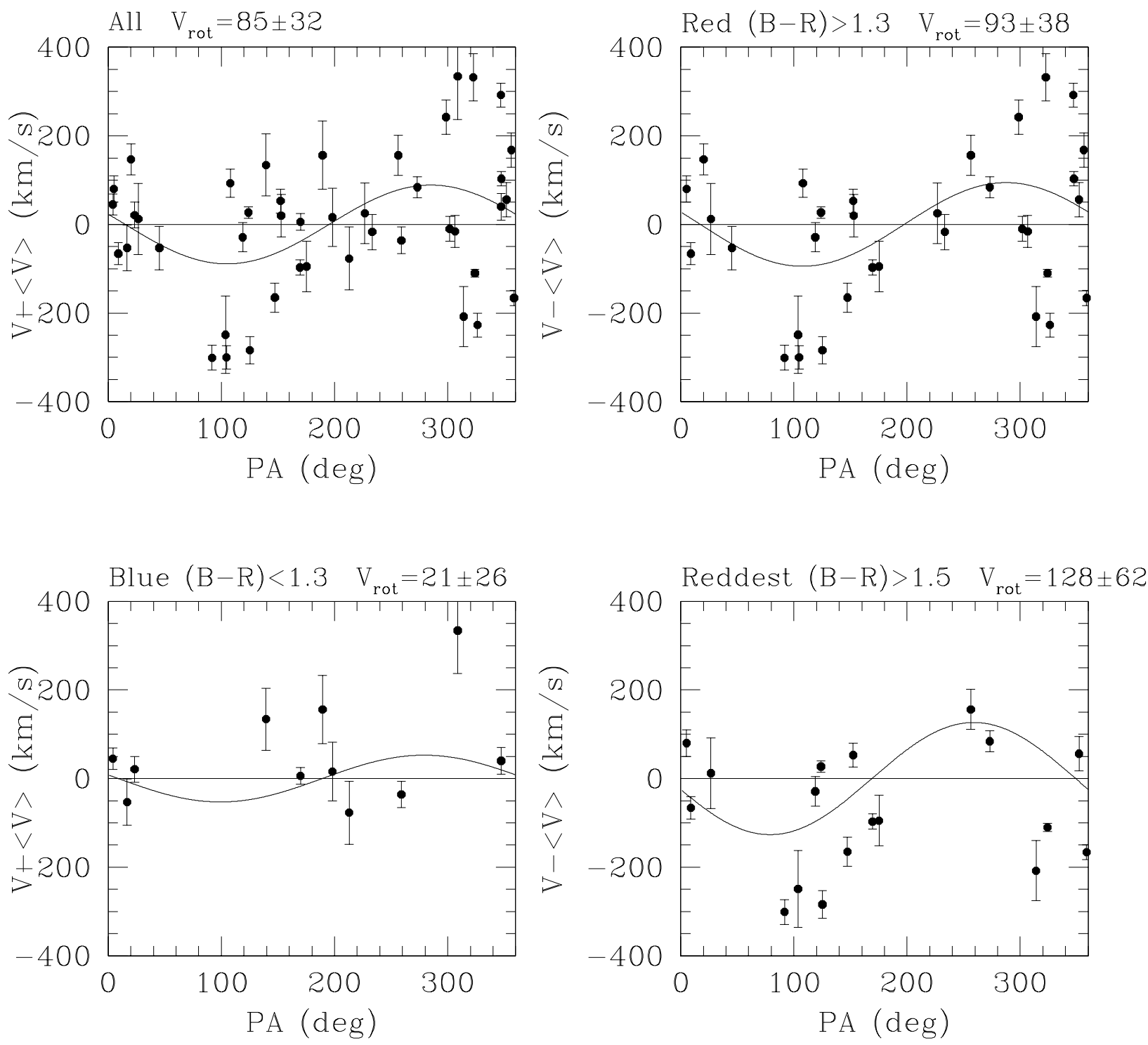

Fig. 9.- Quantitative assessment of rotation in M81 globular cluster sub-samples based on color. Panel $a$ shows rotation for the full range of colors. The rotation velocities were derived using the method of Pryor \& Meylan (1993). The vertical axis is the cluster radial velocity relative to the mean velocity of the full sample. The horizontal axis is the position angle between a cluster and the galaxy center, where a PA of 0 degrees is directly north-south and positive PA is east of north. The best fitting sinusoids to these data represent solid-body rotation and place a lower limit on the rotation velocity of each sample. Rotation is strongly suggested in both of the red, metal-rich sub-samples. The reddest sub-sample also has the lowest velocity dispersion (see Table 7). The blue, metal-poor sub-sample shows no significant evidence for rotation. 

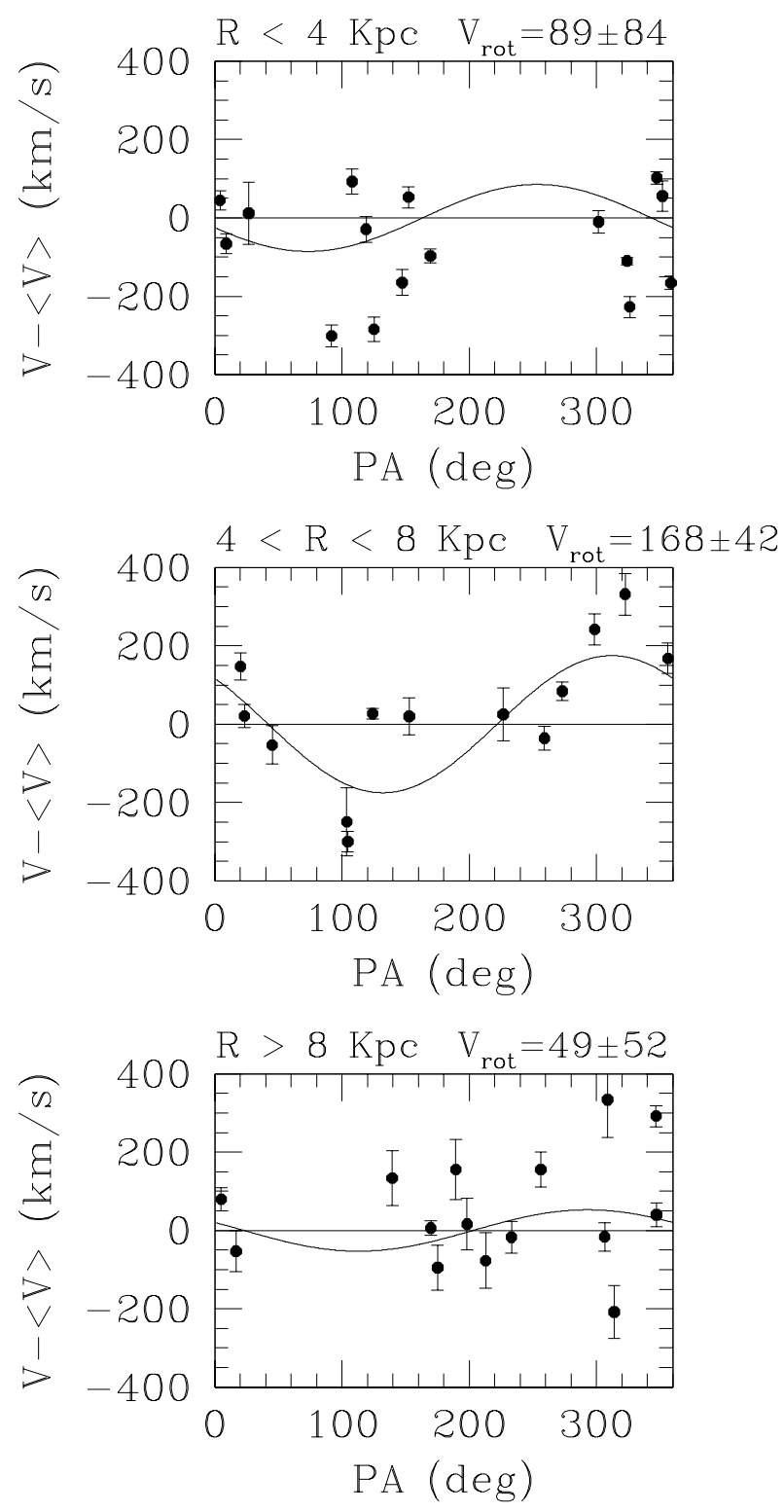

Fig. 10.- Quantitative assessment of rotation in M81 globular cluster sub-samples based on projected galactocentric radius. Only the sub-sample of clusters with intermediate projected galactocentric radii show evidence for rotation. See Section 4.1. 


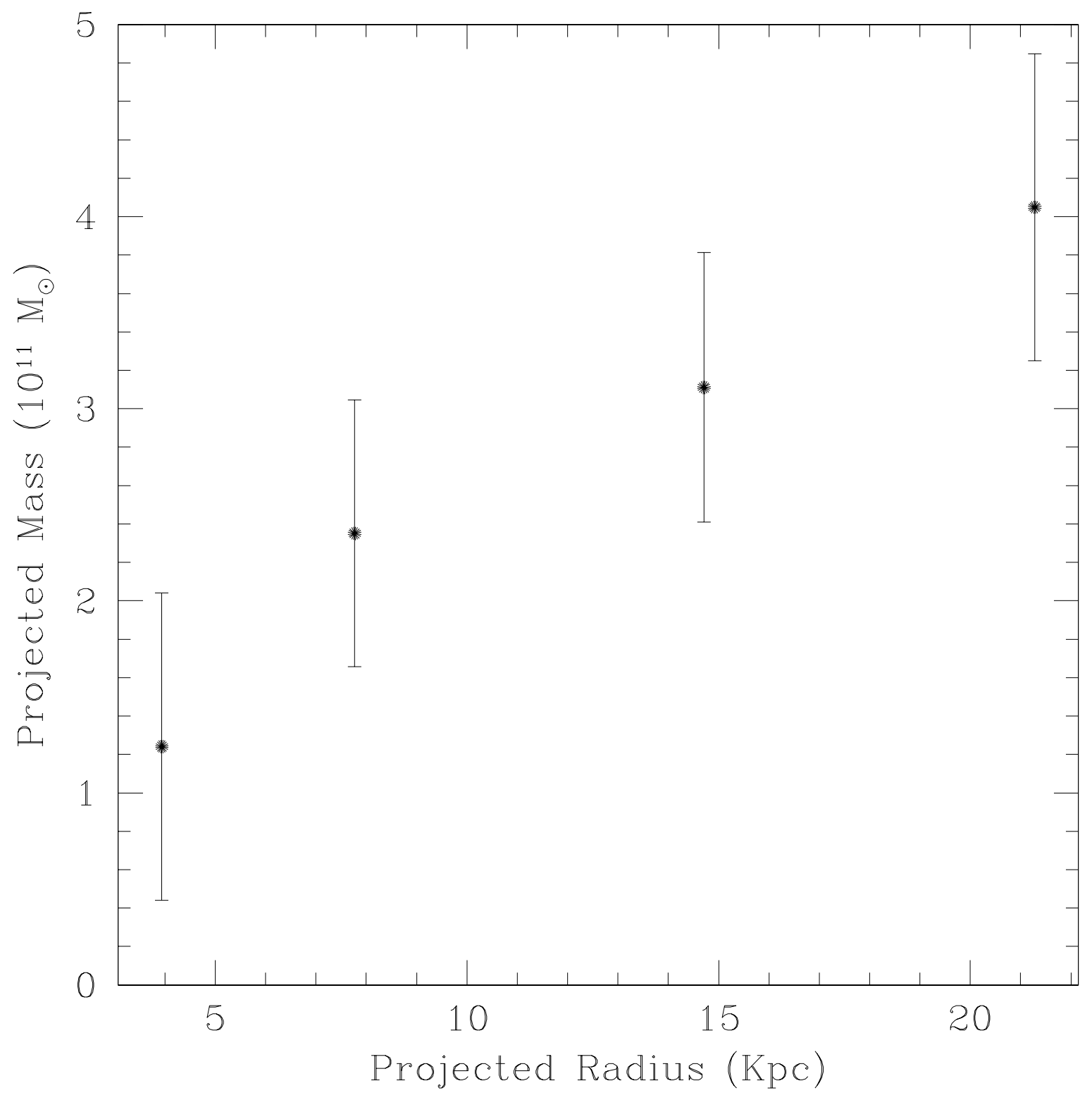

Fig. 11. - Projected mass vs. projected galactocentric radius for M81, derived using radial velocities of M81 globular clusters (see Section 4.2). The increase in mass at large radius is consistent with the presence of a dark-matter halo. 